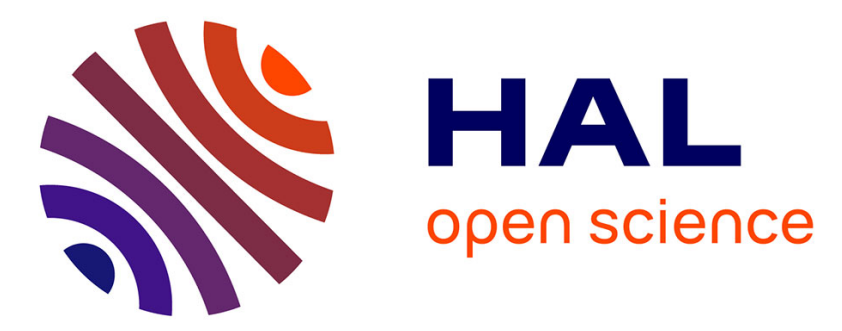

\title{
Coupling schemes for the FSI forward prediction challenge: comparative study and validation
}

Mikel Landajuela, Marina Vidrascu, Dominique Chapelle, Miguel Angel Fernández

\section{- To cite this version:}

Mikel Landajuela, Marina Vidrascu, Dominique Chapelle, Miguel Angel Fernández. Coupling schemes for the FSI forward prediction challenge: comparative study and validation. International Journal for Numerical Methods in Biomedical Engineering, 2017, 33 (4), pp.e02813. 10.1002/cnm.2813 . hal01239931v2

\section{HAL Id: hal-01239931 \\ https://hal.inria.fr/hal-01239931v2}

Submitted on 16 Apr 2016

HAL is a multi-disciplinary open access archive for the deposit and dissemination of scientific research documents, whether they are published or not. The documents may come from teaching and research institutions in France or abroad, or from public or private research centers.
L'archive ouverte pluridisciplinaire HAL, est destinée au dépôt et à la diffusion de documents scientifiques de niveau recherche, publiés ou non, émanant des établissements d'enseignement et de recherche français ou étrangers, des laboratoires publics ou privés. 
Coupling schemes for the FSI forward prediction challenge: comparative study and validation

Mikel Landajuela, Marina Vidrascu, Dominique Chapelle, Miguel A. Fernández

RESEARCH 



\title{
inzián
}

\section{Coupling schemes for the FSI forward prediction challenge: comparative study and validation}

\author{
Mikel Landajuela非 Marina Vidrascu*†, Dominique Chapellet \\ Miguel A. Fernández ${ }^{* \dagger}$ \\ Project-Teams M3DISIM \& REO
}

Research Report $\mathrm{n}^{\circ} \mathrm{RR}-8824$ - December 2015 - 31 pages

\begin{abstract}
This paper presents a numerical study in which several partitioned solution procedures for incompressible fluid-structure interaction are compared and validated against the results of an experimental FSI benchmark. The numerical methods discussed cover the three main families of coupling schemes: strongly coupled, semi-implicit and loosely coupled. Very good agreement is observed between the numerical and experimental results. The comparisons confirm that strong coupling can be efficiently avoided, via semi-implicit and loosely coupled schemes, without compromising stability and accuracy.
\end{abstract}

Key-words: fluid-structure interaction, incompressible fluid, non-linear elastodynamics, Reissner-Mindlin shell, fractional-step method, splitting schemes, partitioned algorithm, experimental data.

* Inria, 75012 Paris, France

† Sorbonne Universités, UPMC Université Paris 6, Laboratoire Jacques-Louis Lions, 75005 Paris, France

¥ Inria and Université Paris-Saclay, 91120 Palaiseau, France

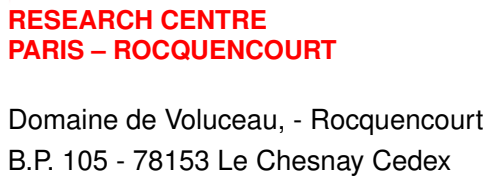




\section{Schémas de couplage pour le challenge FSI: étude comparative et validation}

Résumé : Cet article présente une étude numérique dans laquelle plusieurs algorithmes partitionnés pour l'interaction fluide-structure sont comparés et validés avec des résultats expérimentaux. Les méthodes numériques discutées couvrent les trois familles principales de schémas de couplage: fortement couplés, semi-implicites et faiblement couplés. Un très bon accord est obtenu entre les résultats numériques et expérimentaux. Les comparaisons confirment que le couplage fort peut être contourné efficacement, par l'intermédiaire de schémas semi-implicites et faiblement couplés, sans compromettre la stabilité et la précision.

Mots-clés : interaction fluide-structure, fluide incompressible, élastodynamique nonlinéaire, coque Reissner-Mindlin, méthode à pas fractionnaire, schéma de couplage, algorithme partitionné, données expérimentales. 


\section{Introduction}

Incompressible fluid-structure interaction (FSI), that is to say, the coupling of an incompressible fluid with a deformable solid, is a multi-physic problem omnipresent in nature and in many engineering fields (see, e.g., [54, 75, 27, 64, 66, 81, 45]). The structure is deformed under the action of the fluid and the fluid flow is perturbed by the moving solid.

A fundamental difficulty that has to be faced when solving this coupled problem is related to the extreme stiff nature of the kinematic-dynamic coupling (see, e.g., [50, 20, 38, 78, ). In short, the stability of naive fluid-solid splitting schemes is dictated by the amount of added-mass in the system, rather than by the discretization parameters. In addition, the stiffness of the coupling can also harm the efficiency of (partitioned) iterative solution procedures.

Since the beginning of this century, the development and analysis of numerical methods for FSI overcoming these issues has been a very active research field and the subject of numerous studies (see, e.g., 79, 29, 46, 24, for recent reviews).

A natural classification of FSI numerical methods can be made in terms of the underlying coupling scheme, that is, the time-semidiscrete treatment of the kinematic-dynamic coupling. Strongly coupled schemes treat these interface conditions in a fully implicit fashion (see, e.g., 73. 5. 74, 62, 52]). This guarantees stability and optimal accuracy, but with the outcome of solving a computationally demanding heterogeneous system at each time-step. The solution procedures for these coupled systems typically fall into one of the following two categories: partitioned and monolithic. Partitioned algorithms capitalize on the heterogenous nature of the system via separate solutions of the fluid and solid subsystems, with appropriate exchanges on the interface (see, e.g., [33, 5, 25, 6, 79, 9, 63]). Monolithic methods, on the contrary, solve the heterogeneous FSI problem as a single system of equations, which requires suitable preconditioning (see, e.g., [7, 69, 40, 23, 61, 68]). The intrinsic modularity of a partitioned solution procedure enables the reuse of independent fluid and solid solvers. This advantage comes however at a price, superior efficiency with respect to a monolithic approach is not necessarily guaranteed (see, e.g., [7, 40]).

Computational complexity can be significantly reduced via an explicit-implicit discretization of the kinematic-dynamic interface coupling. These approaches, often referred to in the literature as semi-implicit coupling schemes, use a fractional-step time-discretization in either the fluid (see, e.g., 32, 67, 8, 2, 44) or the solid (see, e.g., 44, 30, 16, 55, 17]) subsystems. The explicit part of the coupling reduces computational complexity, whereas the implicit one guarantees stability.

Finally, loosely coupled schemes (also known as explicit coupling schemes) fully split the fluid and solid time-steppings through specific explicit discretizations of the interface conditions (see, e.g., [18, 19, 31, 35, 13, 36, 37]). The resulting solution procedures are naturally partitioned and, a priori, the least computationally expensive. Numerical stability with these methods has been for years an open problem (see [18]). Accuracy can also be an issue, particularly in the case of the coupling with thick-walled solid models (see, e.g., [31, 34]).

Numerical methods for FSI are generally validated and compared between them using reference synthetic data, that is, generated via numerical experiments (see, e.g., [48, 77, 29, 24]). Comparisons between numerical and experimental results are rare in the FSI literature, and often limited to a single numerical method (see 66, 60, 59, 15]).

The main objective of this paper is to compare and validate some of the aforementioned solution algorithms within the framework of the FSI experimental benchmark presented in 39 . We consider an archetypal sample of state-of-the-art numerical methods covering the above three categories of coupling schemes. All the solution procedures discussed are partitioned and, from the coupling algorithm standpoint, parameter free.

The paper is organized as follows. Section 2 presents the mathematical models and for- 
mulations considered in this work. The numerical algorithms used to simulate the benchmark experiments are described in Section 3. In Section 4, the numerical results are discussed and compared with the experimental data. Finally, a summary of the conclusions and some directions of further investigation are drawn in Section 5.

\section{Problem setting}

This section presents the different mathematical models considered in this work. Full details on the experimental setup are given in 39. The fluid, an aqueous glycerol solution, is modeled by the incompressible Navier-Stokes equations in ALE (arbitrary Lagrangian-Eulerian) formulation. The solid filament, made of silicone, is described by a non-linear viscoelastic (3D or shell) model.

\subsection{Geometry}

As reference configuration for the coupled system, we consider the domain $\Omega^{\mathrm{f}} \cup \Omega^{\mathrm{s}}$ depicted in Figure 1(left). For all $t \in \mathbb{R}^{+}$, the current configuration of the solid is denoted by $\Omega^{\mathrm{s}}(t)$, whereas

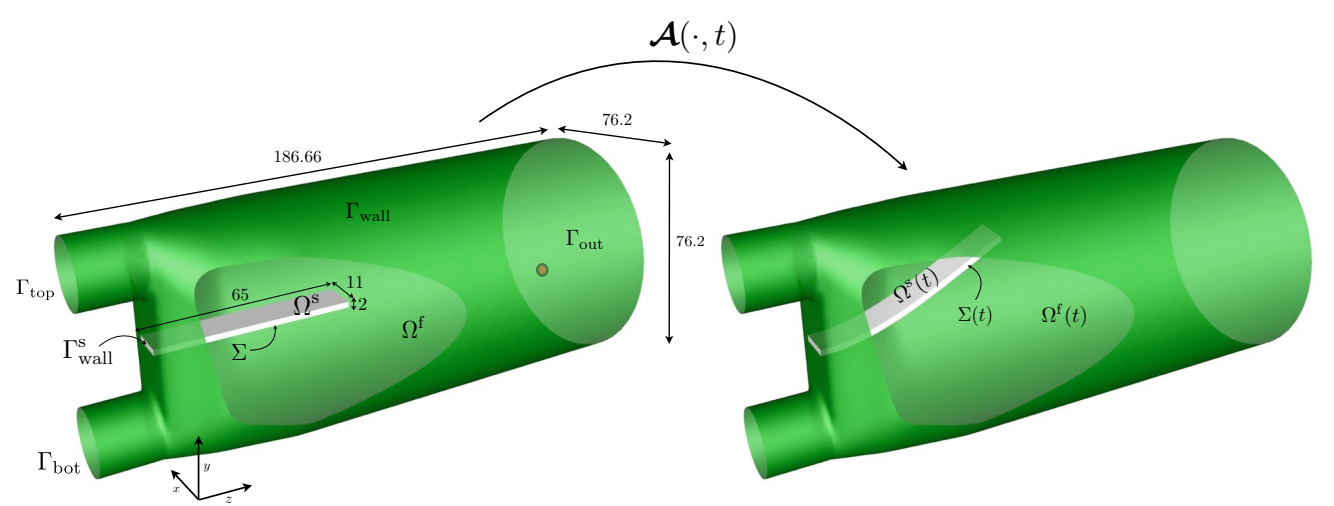

Figure 1: Reference and current geometrical configurations. Lengths are given in millimetres.

the fluid is supposed to fill the moving control volume $\Omega^{\mathrm{f}}(t)$. We denote by $\Sigma(t) \stackrel{\text { def }}{=} \partial \Omega^{\mathrm{f}}(t) \cap \partial \Omega^{\mathrm{s}}(t)$ the current configuration of the fluid-structure interface. Correspondingly, $\Sigma \stackrel{\text { def }}{=} \partial \Omega^{\mathrm{f}} \cap \partial \Omega^{\mathrm{s}}$ stands for the reference fluid-structure interface. The remaining parts of the fluid and solid boundaries $\partial \Omega^{\mathrm{f}} \backslash \Sigma$ and $\partial \Omega^{\mathrm{s}} \backslash \Sigma$ are assumed to be independent of time and partitioned as

$$
\partial \Omega^{\mathrm{f}}=\Gamma_{\text {top }} \cup \Gamma_{\text {bot }} \cup \Gamma_{\text {out }} \cup \Gamma_{\text {wall }} \cup \Sigma, \quad \partial \Omega^{\mathrm{s}}=\Gamma_{\text {wall }}^{\mathrm{s}} \cup \Sigma,
$$

respectively. In the succeeding text, $\boldsymbol{n}$ and $\boldsymbol{n}^{\text {s }}$ refer to the outward normal vectors, on either the current or reference configuration, of the fluid and solid boundaries respectively. Furthermore, for a given vector field $\boldsymbol{v}$ defined on the surface $\Sigma$, the symbols $\boldsymbol{v}_{\perp} \stackrel{\text { def }}{=}(\boldsymbol{v} \cdot \boldsymbol{n}) \boldsymbol{n}$ and $\boldsymbol{v}_{\|} \stackrel{\text { def }}{=} \boldsymbol{v}-\boldsymbol{v}_{\perp}$ denote, respectively, the normal and tangential components of $\boldsymbol{v}$.

The moving fluid domain $\Omega^{\mathrm{f}}(t)$ is parametrized as $\Omega^{\mathrm{f}}(t)=\mathcal{A}\left(\Omega^{\mathrm{f}}, t\right)$ where $\mathcal{A}: \Omega^{\mathrm{f}} \times \mathbb{R}^{+} \rightarrow \mathbb{R}^{3}$ stands for the ALE map (see Figure 1), given by the relation $\mathcal{A}=\boldsymbol{I}_{\Omega^{\mathrm{f}}}+\boldsymbol{d}^{\mathrm{f}}$ in terms of the fluid domain displacement $\boldsymbol{d}^{\mathrm{f}}: \Omega^{\mathrm{f}} \times \mathbb{R}^{+} \rightarrow \mathbb{R}^{3}$. The symbol $\boldsymbol{w} \stackrel{\text { def }}{=} \partial_{t} \mathcal{A}=\partial_{t} \boldsymbol{d}^{\mathrm{f}}$ denotes the fluid domain velocity, $\boldsymbol{F} \stackrel{\text { def }}{=} \boldsymbol{\nabla} \mathcal{A}$ the gradient of deformation and $J \stackrel{\text { def }}{=} \operatorname{det} \boldsymbol{F}$ the Jacobian. 
Remark 2.1. Note that a physical field defined in the reference fluid domain $\Omega^{\mathrm{f}}$, is evaluated in the current fluid domain, $\Omega^{\mathrm{f}}(t)$, by composition with $\mathcal{A}^{-1}(\cdot, t)$. In order to ease the presentation, this change of variable will be suppressed in the following.

\subsection{The coupled fluid-structure problem}

We will consider both cases in which the solid filament is modeled as a thick-walled (3D) solid or as a thin-walled (shell) solid.

\subsubsection{Coupling with 3D solid model}

The resulting coupled problem reads as follows: find the fluid domain displacement $\boldsymbol{d}^{\mathrm{f}}: \Omega^{\mathrm{f}} \times \mathbb{R}^{+} \rightarrow$ $\mathbb{R}^{d}$, the fluid velocity $\boldsymbol{u}: \Omega^{\mathrm{f}} \times \mathbb{R}^{+} \rightarrow \mathbb{R}^{d}$, the fluid pressure $p: \Omega^{\mathrm{f}} \times \mathbb{R}^{+} \rightarrow \mathbb{R}$, the structure displacement $\boldsymbol{d}: \Omega^{\mathrm{s}} \times \mathbb{R}^{+} \rightarrow \mathbb{R}^{d}$ and the structure velocity $\dot{\boldsymbol{d}}: \Omega^{\mathrm{s}} \times \mathbb{R}^{+} \rightarrow \mathbb{R}^{d}$, such that for all $t>0$

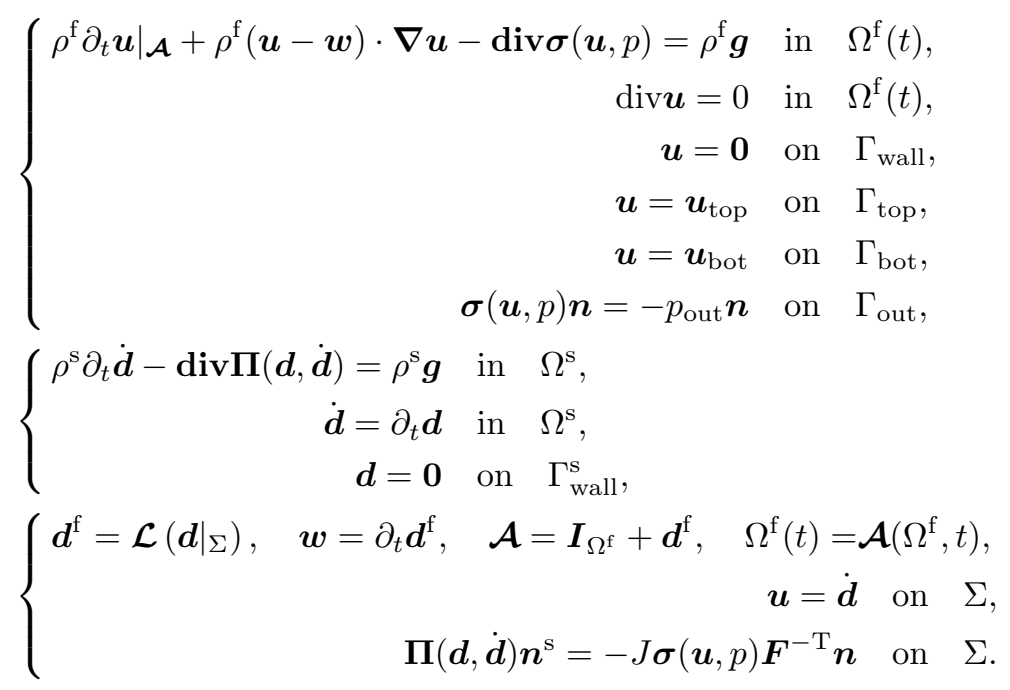

The relation $(3)_{1}$ guarantees the geometrical compatibility between the fluid and solid domains. The operator $\mathcal{L}$ represents a suitable lifting of $\left.\boldsymbol{d}\right|_{\Sigma}$ over $\Omega^{\mathrm{f}}$, with homogeneous boundary conditions on $\partial \Omega^{\mathrm{f}} \backslash \Sigma$ (see Section 3.3). The remaining interface conditions $(3)_{2,3}$ enforce, respectively, the so-called interface kinematic and dynamic coupling.

The symbols $\rho^{\mathrm{f}}$ and $\rho^{\mathrm{s}}$ stand, respectively, for the fluid and solid densities, $\left.\partial_{t}\right|_{\mathcal{A}}$ for the ALE time derivative, $\boldsymbol{g}$ for the acceleration due to gravity and $\boldsymbol{\sigma}(\boldsymbol{u}, p) \stackrel{\text { def }}{=}-p \boldsymbol{I}+2 \mu \boldsymbol{\varepsilon}(\boldsymbol{u})$ for the fluid Cauchy stress tensor, with $\mu$ the fluid dynamic viscosity and $\boldsymbol{\varepsilon}(\boldsymbol{u}) \stackrel{\text { def }}{=} \frac{1}{2}\left(\boldsymbol{\nabla} \boldsymbol{u}+\boldsymbol{\nabla} \boldsymbol{u}^{\mathrm{T}}\right)$ the strain rate tensor. The velocity profiles, $\boldsymbol{u}_{\text {top }}$ and $\boldsymbol{u}_{\text {bot }}$, and the pressure field, $p_{\text {out }}$, are given data on $\Gamma_{\text {top }}, \Gamma_{\text {bot }}$ and $\Gamma_{\text {out }}$, respectively. The first Piola-Kirchhoff stress tensor of the structure $\Pi(\boldsymbol{d}, \dot{\boldsymbol{d}})$ is assumed to be given by the relation

$$
\Pi(\boldsymbol{d}, \dot{\boldsymbol{d}}) \stackrel{\text { def }}{=} \boldsymbol{\Lambda}(\boldsymbol{d})+\eta \partial_{d} \boldsymbol{\Lambda}(\mathbf{0}) \dot{d}
$$

where the first and second terms, respectively, describe the elastic and viscous behavior of the solid. Here, the symbol $\boldsymbol{\partial}_{\boldsymbol{d}} \boldsymbol{\Lambda}(\mathbf{0})$ denotes the Fréchet derivative of $\boldsymbol{\Lambda}$ at $\mathbf{0}$ and $\eta>0$ is the damping coefficient. 
Finally, the coupled system (1)-(3) is complemented with the following initial conditions

$$
\boldsymbol{u}(0)=\mathbf{0}, \quad \boldsymbol{d}(0)=\boldsymbol{d}_{0}, \quad \dot{\boldsymbol{d}}(0)=\mathbf{0}
$$

where $\boldsymbol{d}_{0}$ denotes the displacement undergone by the solid in its hydrostatic equilibrium, viz., solution of (1)-(3) with $\boldsymbol{u}_{\text {in }}^{\text {top }}=\boldsymbol{u}_{\text {in }}^{\text {bot }}=\mathbf{0}$ (no flow conditions).

\subsubsection{Coupling with thin-walled solid model}

We consider a shell model of Reissner-Mindlin type with reference configuration given by the mid-surface of $\Omega^{\mathrm{s}}$ (see Figure 2). We will identify this mid-surface with the fluid-structure interface $\Sigma$ and neglect shell thickness effects in the interface coupling. This is a widely used

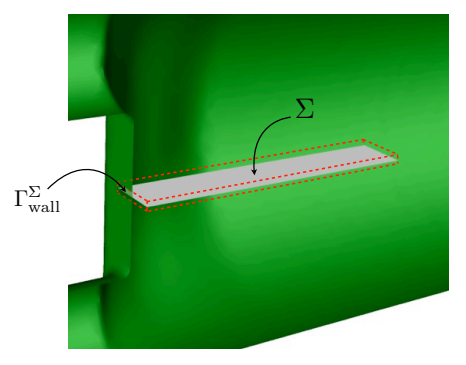

Figure 2: Shell mid-surface and virtual volume of displaced fluid.

modeling simplification when a thin-walled solid is coupled with a 3D medium (see, e.g., 222).

We denote by $\Gamma_{\text {wall }}^{\Sigma}$ the part of the boundary $\partial \Sigma$ that lies in $\Gamma_{\text {wall }}^{\mathrm{s}}$. The interface $\Sigma$ is assumed to be oriented by a unit surface normal vector field (pointing upwards) denoted by $\boldsymbol{n}^{\mathrm{s}}$. This defines a positive and a negative side in the fluid domain $\Omega^{\mathrm{f}}$, with respective unit normals $\boldsymbol{n}^{+} \stackrel{\text { def }}{=} \boldsymbol{n}^{\mathrm{s}}$ and $\boldsymbol{n}^{-} \stackrel{\text { def }}{=}-\boldsymbol{n}^{\mathrm{s}}$ on $\Sigma$. For a given continuous scalar or tensorial field $f$ defined in $\Omega^{\mathrm{f}}$ (possibly discontinuous across $\Sigma$ ), we define its sided-restrictions to $\Sigma$, denoted by $f^{+}$and $f^{-}$, as $f^{+}(\boldsymbol{x}) \stackrel{\text { def }}{=} \lim _{\xi \rightarrow 0^{+}} f\left(\boldsymbol{x}+\xi \boldsymbol{n}^{+}\right)$and $f^{-}(\boldsymbol{x}) \stackrel{\text { def }}{=} \lim _{\xi \rightarrow 0^{+}} f\left(\boldsymbol{x}+\xi \boldsymbol{n}^{-}\right)$for all $\boldsymbol{x} \in \Sigma$. We also define the following jumps across the interface $\Sigma$ :

$$
\llbracket f \rrbracket \stackrel{\text { def }}{=} f^{+}-f^{-}, \quad \llbracket f \boldsymbol{n} \rrbracket \stackrel{\text { def }}{=} f^{+} \boldsymbol{n}^{+}+f^{-} \boldsymbol{n}^{-} .
$$

The shell kinematics are entirely characterized by the displacement field of the mid-surface $\boldsymbol{d}$ and the field of director vectors in the deformed configuration $\boldsymbol{a}$, i.e., the unit vectors that underlie the material lines originally orthogonal to the midsurface in the undeformed configuration. We recall that Reissner-Mindlin kinematics assume that such material lines, originally aligned with $\boldsymbol{n}$, remain straight and preserve their length in the deformation (see, e.g., [26, 21]).

In this framework, the resulting coupled fluid-structure problem reads as follows: find the fluid domain displacement $\boldsymbol{d}^{\mathrm{f}}: \Omega^{\mathrm{f}} \times \mathbb{R}^{+} \rightarrow \mathbb{R}^{d}$, the fluid velocity $\boldsymbol{u}: \Omega^{\mathrm{f}} \times \mathbb{R}^{+} \rightarrow \mathbb{R}^{d}$, the fluid pressure $p: \Omega^{\mathrm{f}} \times \mathbb{R}^{+} \rightarrow \mathbb{R}$, the solid mid-surface displacement $\boldsymbol{d}: \Sigma \times \mathbb{R}^{+} \rightarrow \mathbb{R}^{3}$ and the director 
vector $\boldsymbol{a}: \Sigma \times \mathbb{R}^{+} \rightarrow \mathbb{R}^{3}$ of unit length $|\boldsymbol{a}|=1$, such that (1) and

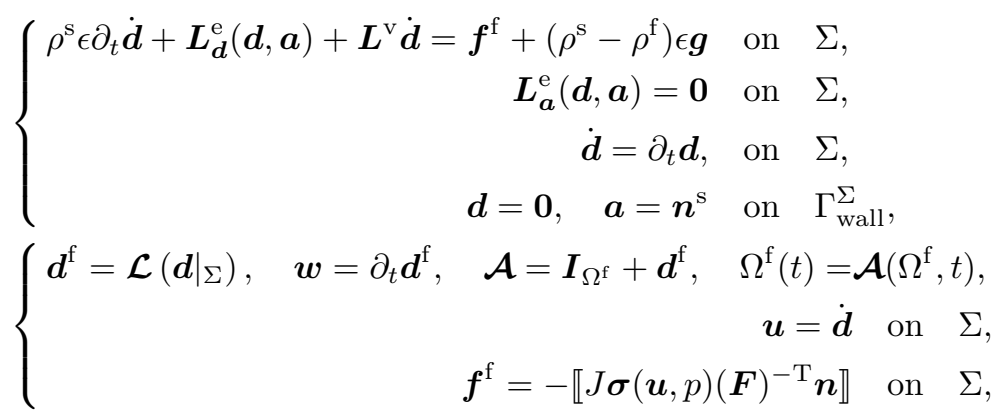

hold for all $t>0$. Here, the surface operators $\boldsymbol{L}_{\boldsymbol{d}}^{\mathrm{e}}$ and $\boldsymbol{L}_{\boldsymbol{a}}^{\mathrm{e}}$ govern the elastic behaviour of the shell, with $\boldsymbol{L}_{\boldsymbol{a}}^{\mathrm{e}}=\mathbf{0}$ representing, in particular, the equilibrium of bending moments and shear stresses. Physical damping is modeled through the linear term $\boldsymbol{L}^{\mathrm{v}} \dot{\boldsymbol{d}} \stackrel{\text { def }}{=} \eta \boldsymbol{\partial}_{\boldsymbol{d}} \boldsymbol{L}^{\mathrm{e}}(\mathbf{0}, \mathbf{0}) \dot{\boldsymbol{d}}$, with $\eta>0$. Rotational inertia is neglected, and gravitational effects are taken into account through the surface force $\rho^{\mathrm{s}} \epsilon \boldsymbol{g}$. Note that, since the solid is geometrically assimilated to a surface, it does not displace any fluid and, hence, no buoyancy force is undergone by the structure. This is corrected by including the buoyancy force in an approximate static manner, namely, by adding the Archimedes surface force term $-\rho^{\mathrm{f}} \epsilon \boldsymbol{g}$.

Remark 2.2. The jump of the hydro-dynamic stress through the immersed solid results in pressure and velocity solutions that are, respectively, strongly and weakly (gradient) discontinuous along the moving fluid-structure interface. In the discrete ALE setting (i.e., with fitted fluidsolid meshes), these discontinuities can be naturally included in the finite element approximation spaces (see Section 3.2).

\subsection{Fluid}

Two different experimental phases, corresponding to a stationary or a pulsatile flow regime, are considered (see [39]). In [1] we take $\boldsymbol{g}=-g \mathbf{e}_{y}$ with $g \stackrel{\text { def }}{=} 9.80665 \cdot 10^{3} \mathrm{~mm} \mathrm{~s}^{-2}$. An hydrostatic pressure profile of the form

$$
p_{\text {out }}(y)=p_{0}-\rho^{\mathrm{f}} g\left(y-y_{0}\right),
$$

is prescribed on the outlet boundary $\Gamma_{\text {out }}$, where $p_{0}=1782.7 \mathrm{~Pa}$ is a given pressure value measured at point $(30.00,-26.38,160.20)$ (the red point in Figure 1).

\subsubsection{Phase I experiment}

During this phase, the fluid is pumped in the domain with a constant flow rate. The $z$ components of the inlet velocity profiles $\boldsymbol{u}_{\text {top }}$ and $\boldsymbol{u}_{\text {bot }}$ are parabolic with a peak velocity of $630 \mathrm{~mm} \mathrm{~s}^{-1}$ and $615 \mathrm{~mm} \mathrm{~s}^{-1}$, respectively. The other components are set to zero. The density and dynamic viscosity of the fluid are given in Table 1 . These values were measured with the glycerol at a temperature of $T=23.6^{\circ} \mathrm{C}$ (see [39]).

\subsubsection{Phase II experiment}

In the second phase of the experiment, the fluid is pumped with a pulsatile flow rate of frequency

$\mathrm{RR} \mathrm{n}^{\circ} \mathrm{RR}-8824$ 


\begin{tabular}{|c|c|}
\hline$\rho^{\mathrm{f}}$ & $\mu$ \\
\hline $1.1633 \cdot 10^{-3} \mathrm{~g} \mathrm{~mm}^{-3}$ & $12.5 \cdot 10^{-3} \mathrm{~g} \mathrm{~mm}^{-1} \mathrm{~s}^{-1}$ \\
\hline
\end{tabular}

Table 1: Fluid physical parameters (Phase I experiment).

$1 / 6 s^{-1}$. The profiles of the $x-, y$ - and $z$-components of $\boldsymbol{u}_{\text {top }}$ are parabolic with peak values over time given in Figure 3 . The $z$ - and $x$-components of the bottom inlet velocity $\boldsymbol{u}_{\text {bot }}$ are the same as in $\boldsymbol{u}_{\text {top }}$, but its $y$-component is set to zero. The density and dynamic viscosity of the fluid are given in Table 2. In this case, the measurements were obtained for the glycerol at $T=22{ }^{\circ} \mathrm{C}($ see 39$]$ ).

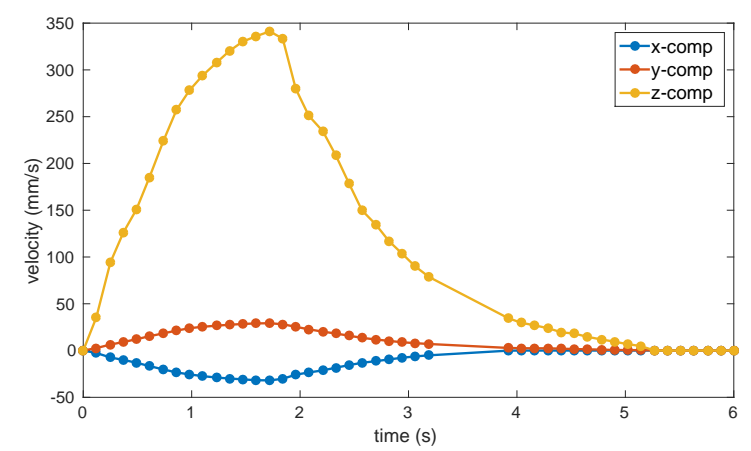

Figure 3: Measured peak values in the $x-, y-$ and $z$-components of the inlet velocity $\boldsymbol{u}_{\text {top }}$.

\begin{tabular}{|c|c|}
\hline$\rho^{\mathrm{f}}$ & $\mu$ \\
\hline $1.164 \cdot 10^{-3} \mathrm{~g} \mathrm{~mm}$ & -3 \\
\hline
\end{tabular}

Table 2: Fluid physical parameters (Phase II experiment).

\subsection{Solid}

The silicone filament is assumed to be homogeneous with density $\rho^{\mathrm{s}}=1.063 \cdot 10^{-3} \mathrm{~g} \mathrm{~mm}^{-3}$. For the discussion of the elastic constitutive model, we classically introduce the gradient of deformation $\boldsymbol{F}^{\mathrm{s}} \stackrel{\text { def }}{=} \boldsymbol{I}+\boldsymbol{\nabla} \boldsymbol{d}$, the Green-Lagrange strain tensor $\boldsymbol{E} \stackrel{\text { def }}{=} \frac{1}{2}\left(\left(\boldsymbol{F}^{\mathrm{s}}\right)^{\mathrm{T}} \boldsymbol{F}^{\mathrm{s}}-\boldsymbol{I}\right)$ and the second Piola-Kirchhoff stress tensor $\boldsymbol{\Sigma} \stackrel{\text { def }}{=}\left(\boldsymbol{F}^{\mathrm{s}}\right)^{-1} \boldsymbol{\Lambda}$. The elastic properties of the solid are described with a Saint Venant-Kirchhoff material, so that

$$
\boldsymbol{\Sigma}=L_{1}(\operatorname{tr} \boldsymbol{E}) \boldsymbol{I}+2 L_{2} \boldsymbol{E} .
$$

Here, $L_{1}, L_{2}>0$ denote the first and second Lamé coefficients of the material, respectively.

Owing to (4) and the above identities, we finally get that

$$
\Pi(d, \dot{d})=F^{\mathrm{s}} \boldsymbol{\Sigma}(\boldsymbol{d})+\eta \partial_{d} \boldsymbol{\Sigma}(\mathbf{0}) \dot{d}
$$


As usual, the elastic properties of the silicone are given in terms of its Young modulus $E$ and Poisson ratio $\nu$. These quantities are related to the Lamé coefficients through the relations

$$
L_{1} \stackrel{\text { def }}{=} \frac{E \nu}{(1-2 \nu)(1+\nu)}, \quad L_{2} \stackrel{\text { def }}{=} \frac{E}{2(1+\nu)} .
$$

\subsubsection{Solid parameters estimation}

Young's modulus $E$ and the Poisson ratio $\nu$ for the silicone were determined from the data obtained in the uniaxial traction test (see 39]). For each loading step we computed the component of the second Piola-Kirchhoff stress tensor in the traction direction, i.e., along the $z$-axis, the corresponding component of the Green-Lagrange strain tensor, and the transverse component of this same strain tensor. Retaining only the first 20 loading steps, linear regression was performed to estimate Young's modulus and the Poisson ratio, yielding the values given in Table 3 Figure 4 compares the regressions with the experimental points of the complete dataset. We can see that the linear regression is quite accurate for extension rates of up to $25 \%$, which justifies the use of a linear stress-strain law - namely, the Saint Venant-Kirchhoff constitutive equation, also known as generalized Hooke's law - for representing the behaviour of this material in the stress-strain ranges of practical interest in our study.

\begin{tabular}{|c|c|}
\hline$E$ & $\nu$ \\
\hline $2.1626 \cdot 10^{5} P a$ & 0.3151 \\
\hline
\end{tabular}

Table 3: Estimated constitutive parameters (Young's modulus $E$ and Poisson ratio $\nu$ ) based on uniaxial traction data from 39 .

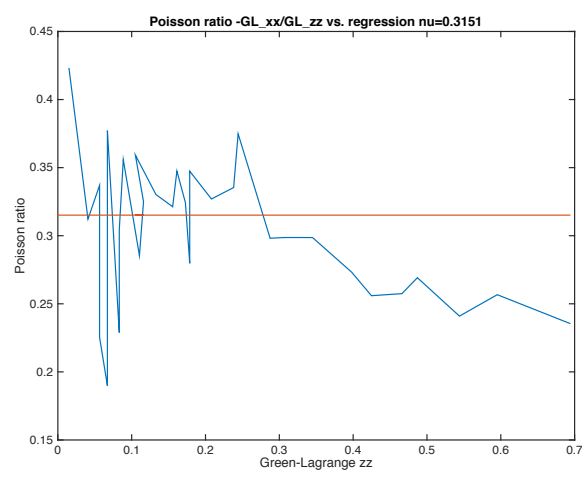

(a)

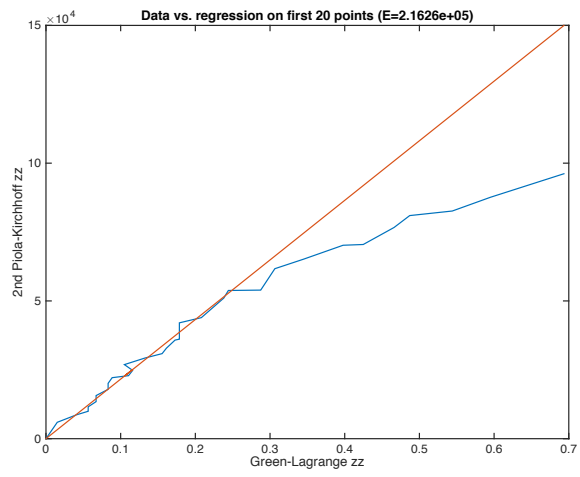

(b)

Figure 4: Poisson ration and Young's modulus regressions.

The damping coefficient $\eta$ has been tuned in order to remove torsional oscillations in the solid not observed during the experiments. The value considered in the numerical results of Section 4 is $\eta=2.5 \cdot 10^{-4} s^{-1}$. 


\section{$3 \quad$ Numerical methods}

This section presents the numerical methods considered in this work for the approximation of the coupled problems (1)-(3) and (1), (6)-(7). Section 3.1 is devoted to the discretization in time and to the fluid-solid splitting schemes. Some ingredients of the spatial discretization are given in Section 3.2. Finally, Section 3.3 presents the mesh update technique in the fluid, namely, the discrete counterpart of the lifting operator $\mathcal{L}$.

\subsection{Time discretization: coupling schemes}

In what follows, the symbol $\tau>0$ denotes the time-step length, $t_{n} \stackrel{\text { def }}{=} n \tau$, for $n \in \mathbb{N}$ and $\partial_{\tau} x^{n} \stackrel{\text { def }}{=}\left(x^{n}-x^{n-1}\right) / \tau$ the first order backward difference in time. For the time discretization of the fluid subsystem (1) we will consider either the following monolithic (semi-implicit) backward Euler scheme:

$$
\left\{\begin{array}{rcc}
\left.\rho^{\mathrm{f}} \partial_{\tau} \boldsymbol{u}^{n}\right|_{\mathcal{A}}+\rho^{\mathrm{f}}\left(\boldsymbol{u}^{n-1}-\boldsymbol{w}^{n}\right) \cdot \boldsymbol{\nabla} \boldsymbol{u}^{n}-\operatorname{div} \boldsymbol{\sigma}\left(\boldsymbol{u}^{n}, p^{n}\right)=\rho^{\mathrm{f}} \boldsymbol{g}, & \text { in } & \Omega^{\mathrm{f}, n}, \\
\operatorname{div} \boldsymbol{u}^{n}=0 & \text { in } & \Omega^{\mathrm{f}, n}, \\
\boldsymbol{u}^{n}=\mathbf{0} & \text { on } & \Gamma_{\text {wall }}, \\
\boldsymbol{u}^{n}=\boldsymbol{u}_{\text {top }}^{n} & \text { on } & \Gamma_{\text {top }}, \\
\boldsymbol{u}^{n}=\boldsymbol{u}_{\text {bot }}^{n} & \text { on } & \Gamma_{\text {bot }}, \\
\boldsymbol{\sigma}\left(\boldsymbol{u}^{n}, p^{n}\right) \boldsymbol{n}=-p_{\text {out }}^{n} \boldsymbol{n} & \text { on } & \Gamma_{\text {out }}
\end{array}\right.
$$

or the following incremental pressure-correction scheme:

1. Fluid convective-viscous substep:

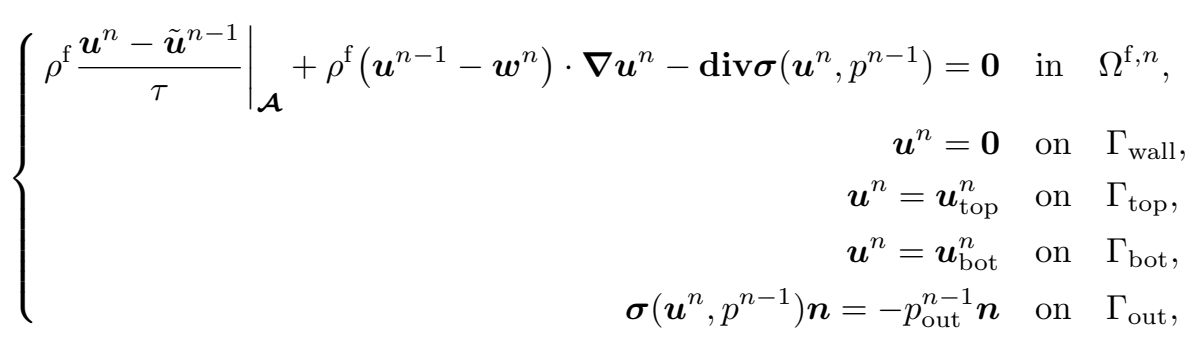

2. Fluid projection substep:

$$
\left\{\begin{aligned}
-\frac{\tau}{\rho^{\mathrm{f}}} \Delta \phi^{n}=-\operatorname{div} \boldsymbol{u}^{n} & \text { in } \quad \Omega^{\mathrm{f}, n} \\
\frac{\tau}{\rho^{\mathrm{f}}} \frac{\partial \phi^{n}}{\partial \boldsymbol{n}}=0 & \text { on } \quad \Gamma_{\text {top }} \cup \Gamma_{\text {bot }}, \\
\phi^{n}=p_{\text {out }}^{n}-p^{n-1} \quad & \text { on } \quad \Gamma_{\text {out }},
\end{aligned}\right.
$$

and thereafter we set $p^{n}=\phi^{n}+p^{n-1}, \tilde{\boldsymbol{u}}^{n}=\boldsymbol{u}^{n}-\frac{\tau}{\rho^{\mathrm{f}}} \boldsymbol{\nabla} \phi^{n}$ in $\Omega^{\mathrm{f}}$.

Note that a standard semi-implicit treatment of the convective term is employed in (8) and in (9), which makes these problems linear. For the solid, we consider a backward Euler time-stepping of the $3 \mathrm{D}$ solid subsystem 2,

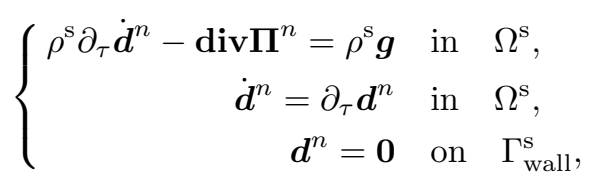


with the notation $\boldsymbol{\Pi}^{n} \stackrel{\text { def }}{=} \boldsymbol{\Lambda}\left(\boldsymbol{d}^{n}\right)+\eta \boldsymbol{\partial}_{\boldsymbol{d}} \boldsymbol{\Lambda}(\mathbf{0}) \dot{\boldsymbol{d}}^{n}$. The shell subsystem 6 is also discretized as

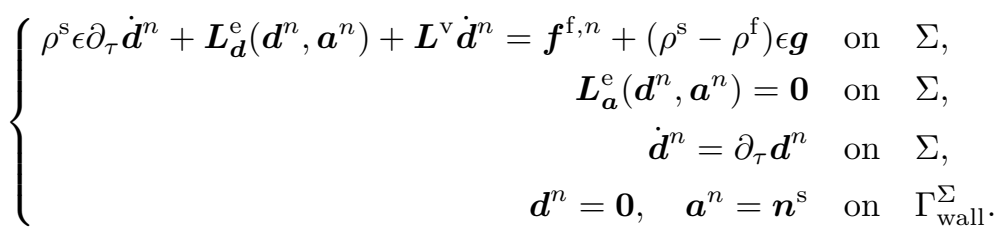

In all the forthcoming solution procedures, we adopt an explicit treatment of the geometric compatibility condition $(3)_{1}$, namely,

$$
\boldsymbol{d}^{\mathrm{f}, n}=\mathcal{L}\left(\left.\boldsymbol{d}^{n-1}\right|_{\Sigma}\right), \quad \boldsymbol{w}^{n}=\partial_{\tau} \boldsymbol{d}^{\mathrm{f}, n}, \quad \mathcal{A}^{n} \stackrel{\text { def }}{=} \boldsymbol{I}_{\Omega^{\mathrm{f}}}+\boldsymbol{d}^{\mathrm{f}, n}, \quad \Omega^{\mathrm{f}, n} \stackrel{\text { def }}{=} \mathcal{A}^{n}\left(\Omega^{\mathrm{f}}\right)
$$

and thereafter we set $\boldsymbol{F}^{n} \stackrel{\text { def }}{=} \boldsymbol{\nabla} \mathcal{A}^{n}$ and $J^{n} \stackrel{\operatorname{def}}{=} \operatorname{det} \boldsymbol{F}^{n}$. This approach significantly reduces computational complexity without compromising stability and accuracy (see, e.g., [72, 32, 5, 74, 62 ). The numerical methods presented in the next paragraphs mainly differ on the time-stepping of the kinematic and dynamic interface conditions $(3)_{2,3}$ or $(7)_{2,3}$.

\subsubsection{Strongly coupled schemes}

The first class of numerical methods considered in this paper performs a fully implicit treatment of the kinematic and dynamic coupling conditions $(3)_{2,3}$ and $(7)_{2,3}$. This yields the time-marching schemes reported in Algorithms 1 and 2 Schemes for which (14) or (15) hold are often referred to in the literature as strongly coupled. Basically, their main advantage lies in the fact that they induce a correct discrete energy balance across the interface and, hence, guarantee energy stability (see, e.g., 72, 5, 74, 62,).

Algorithm 1 Strongly coupled scheme (coupling with 3D solid).

For $n \geq 1$ :

1. Update fluid domain via $(13)$;

2. Find $\boldsymbol{u}^{n}: \Omega^{\mathrm{f}} \rightarrow \mathbb{R}^{3}, p^{n}: \Omega^{\mathrm{f}} \rightarrow \mathbb{R}, \boldsymbol{d}^{n}: \Omega^{\mathrm{s}} \rightarrow \mathbb{R}^{3}$ and $\dot{\boldsymbol{d}}^{n}: \Omega^{\mathrm{s}} \rightarrow \mathbb{R}^{3}$ satisfying (8), (11) and

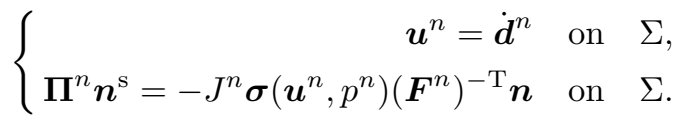

The price to pay for this superior stability is the resolution of a fully-coupled nonlinear system at each time-step (Step 2 of Algorithms 1 and 2), which can be computationally demanding in practice. Owing to the semi-implicit time-stepping in (8), the non-linearity of this coupled system is exclusively due to the non-linear nature of the solid. In this work, we consider the partitioned solution strategy proposed in [33, which involves an interface Newton solver with Dirichlet-Neumann GMRES iterations.

\subsubsection{Projection based semi-implicit coupling schemes}

These schemes avoid strong coupling without compromising stability and accuracy. The fundamental idea (see [32]) consists in combining the fractional-step time-marching in the fluid 
Algorithm 2 Strongly coupled scheme (coupling with thin-walled solid).

For $n \geq 1$ :

1. Update fluid domain via (13);

2. Find $\boldsymbol{u}^{n}: \Omega^{\mathrm{f}} \rightarrow \mathbb{R}^{3}, p^{n}: \Omega^{\mathrm{f}} \rightarrow \mathbb{R}, \boldsymbol{d}^{n}: \Sigma \rightarrow \mathbb{R}^{3}, \dot{\boldsymbol{d}}^{n}: \Sigma \rightarrow \mathbb{R}^{3}$ and $\boldsymbol{a}^{n}: \Sigma \rightarrow \mathbb{R}^{3}$ with $\left|\boldsymbol{a}^{n}\right|=1$, satisfying $(8), 12$ and

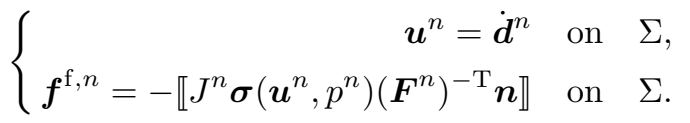

Algorithm 3 Semi-implicit coupling scheme (coupling with 3D solid).

For $n \geq 2$ :

1. Update the fluid domain via 13 ;

2. Explicit step: find $\boldsymbol{u}^{n}: \Omega^{\mathrm{f}} \rightarrow \mathbb{R}^{3}$ satisfying $(9)$ and

$$
\boldsymbol{u}^{n}=\dot{\boldsymbol{d}}^{n-1} \quad \text { on } \quad \Sigma .
$$

3. Implicit step: find $\phi^{n}: \Omega^{\mathrm{f}} \rightarrow \mathbb{R}, \boldsymbol{d}^{n}: \Omega^{\mathrm{s}} \rightarrow \mathbb{R}^{3}$ and $\dot{\boldsymbol{d}}^{n}: \Omega^{\mathrm{s}} \rightarrow \mathbb{R}^{3}$, satisfying 10, 11 and

$$
\left\{\begin{array}{r}
\frac{\tau}{\rho^{\mathrm{f}}} \frac{\partial \phi^{n}}{\partial \boldsymbol{n}}=\left(\boldsymbol{u}^{n}-\dot{\boldsymbol{d}}^{n}\right) \cdot \boldsymbol{n} \quad \text { on } \quad \Sigma \\
\boldsymbol{\Pi}^{n} \boldsymbol{n}^{\mathrm{s}}=-J^{n} \boldsymbol{\sigma}\left(\boldsymbol{u}^{n}, p^{n}\right)\left(\boldsymbol{F}^{n}\right)^{-\mathrm{T}} \boldsymbol{n} \quad \text { on } \quad \Sigma .
\end{array}\right.
$$

(9) - 10 with a specific explicit-implicit treatment of the kinematic and dynamic coupling conditions $(3)_{2,3}$ and $(7)_{2,3}$. The resulting solution procedures are detailed in Algorithms 3 and 4 .

The fluid convective-viscous step $(9)$ is explicitly coupled with the solid, this reduces computational complexity without compromising stability. On the other hand, the solid projection step 10 is implicitly coupled to the solid to avoid added-mass stability issues. Note that this implicit part of the coupling (i.e., Step 3 of Algorithms 3 and 4 is less computationally onerous than Step 2 of Algorithms 1 and 2. In this work, the resulting pressure-solid systems are solved via interface Dirichlet-Neumann GMRES iterations (as for Algorithm 1).

\subsubsection{Explicit coupling schemes}

In this section, we focus on the case of the coupling with a thin-walled solid model, namely, the coupled problem (1), (6)-(7). The methods presented below perform a complete splitting of the fluid and solid time-steppings. The fundamental ingredient in the derivation of these methods (see [31, 35, 37]) is the following interface Robin consistency

$$
\llbracket J \boldsymbol{\sigma}(\boldsymbol{u}, p) \boldsymbol{F}^{-\mathrm{T}} \boldsymbol{n} \rrbracket+\rho^{\mathrm{s}} \epsilon \partial_{t} \boldsymbol{u}=-\boldsymbol{L}_{\boldsymbol{d}}^{\mathrm{e}}(\boldsymbol{d}, \boldsymbol{a})-\boldsymbol{L}^{\mathrm{v}} \dot{\boldsymbol{d}}+\left(\rho^{\mathrm{s}}-\rho^{\mathrm{f}}\right) \epsilon \boldsymbol{g} \quad \text { on } \quad \Sigma,
$$

which can be inferred from $(6)_{1}$ and $(7)_{2,3}$. 
Algorithm 4 Semi-implicit coupling scheme (coupling with thin-walled solid).

For $n \geq 2$ :

1. Update the fluid domain via 13 ;

2. Explicit step: find $\boldsymbol{u}^{n}: \Omega^{\mathrm{f}} \rightarrow \mathbb{R}^{3}$ satisfying $(9)$ and

$$
\boldsymbol{u}^{n}=\dot{\boldsymbol{d}}^{n-1} \quad \text { on } \quad \Sigma .
$$

3. Implicit step: find $\phi^{n}: \Omega^{\mathrm{f}} \rightarrow \mathbb{R}, \boldsymbol{d}^{n}: \Sigma \rightarrow \mathbb{R}^{3}, \dot{\boldsymbol{d}}^{n}: \Sigma \rightarrow \mathbb{R}^{3}$ and $\boldsymbol{a}^{n}: \Sigma \rightarrow \mathbb{R}^{3}$ with $\left|\boldsymbol{a}^{n}\right|=1$, satisfying 10,12 and

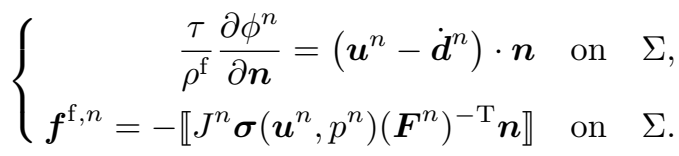

The key idea is hence to perform the fluid-solid splitting by discretizing in time (16) instead of $(7)_{2}$. Then, owing to the dynamic coupling condition $(7)_{3}$, the resulting interface fluid stresses are transferred to the thin-walled solid through the "Neumann"-like relation

$$
\boldsymbol{f}^{\mathrm{f}, n}=-\llbracket J^{n} \boldsymbol{\sigma}\left(\boldsymbol{u}^{n}, p^{n}\right)\left(\boldsymbol{F}^{n}\right)^{-\mathrm{T}} \boldsymbol{n} \rrbracket \quad \text { on } \quad \Sigma .
$$

Robin-Neumann explicit coupling scheme. Following [35], the consistent interface relation (16) is discretized in time as

$$
\begin{aligned}
\llbracket J^{n} \boldsymbol{\sigma}\left(\boldsymbol{u}^{n}, p^{n}\right)\left(\boldsymbol{F}^{n}\right)^{-\mathrm{T}} \boldsymbol{n} \rrbracket+\frac{\rho^{\mathrm{s}} \epsilon}{\tau} \boldsymbol{u}^{n}= & \frac{\rho^{\mathrm{s}} \epsilon}{\tau} \dot{\boldsymbol{d}}^{n-1} \\
& -\boldsymbol{L}_{\boldsymbol{d}}^{\mathrm{e}}\left(\boldsymbol{d}^{n-1}, \boldsymbol{a}^{n-1}\right)-\boldsymbol{L}^{\mathrm{v}} \dot{\boldsymbol{d}}^{n-1}+\left(\rho^{\mathrm{s}}-\rho^{\mathrm{f}}\right) \epsilon \boldsymbol{g} \quad \text { on } \quad \Sigma,
\end{aligned}
$$

which provides an explicit Robin condition for the fluid sub-system (8). Note that the solid inertia is implicitly coupled to the fluid, via the Robin term in the left-hand side of (18), while the solid viscoelastic terms are treated explicitly in $(18)$. The first guarantees (added-mass free) stability whereas the second enables the splitting between the fluid and solid time-steppings.

Owing to (12) and (17), for $n \geq 2$ the Robin condition (18) can be reformulated in a more convenient fashion as follows

$$
\begin{array}{rl}
\llbracket J^{n} \boldsymbol{\sigma}\left(\boldsymbol{u}^{n}, p^{n}\right)\left(\boldsymbol{F}^{n}\right)^{-\mathrm{T}} & \boldsymbol{n} \rrbracket+\frac{\rho^{\mathrm{s}} \epsilon}{\tau} \boldsymbol{u}^{n} \\
& =\frac{\rho^{\mathrm{s}} \epsilon}{\tau}\left(\dot{\boldsymbol{d}}^{n-1}+\tau \partial_{\tau} \dot{\boldsymbol{d}}^{n-1}\right)+\llbracket J^{n-1} \boldsymbol{\sigma}\left(\boldsymbol{u}^{n-1}, p^{n-1}\right)\left(\boldsymbol{F}^{n-1}\right)^{-\mathrm{T}} \boldsymbol{n} \rrbracket \quad \text { on } \quad \Sigma .
\end{array}
$$

The complete explicit coupling scheme is reported in Algorithm 5 .

Fully decoupled scheme. Using the arguments reported in [37, the above explicit Robin-Neumann paradigm can also be effectively combined with the fractional-step fluid time-stepping (9)-(10). The resulting method is presented in Algorithm 6. A salient feature of this solution procedure is that it enables a fully decoupled sequential computation of the whole fluid-solid state: $\boldsymbol{u}^{n}, p^{n}$ and $\left(\boldsymbol{d}^{n}, \boldsymbol{a}^{n}\right)$.

$\mathrm{RR} \mathrm{n}^{\circ} \mathrm{RR}-8824$ 
Algorithm 5 Robin-Neumann explicit coupled scheme.

For $n \geq 2$ :

1. Update the fluid domain via 13 ;

2. Fluid step: find $\boldsymbol{u}^{n}: \Omega^{\mathrm{f}} \rightarrow \mathbb{R}^{3}$ and $p^{n}: \Omega^{\mathrm{f}} \rightarrow \mathbb{R}$ satisfying 8 and

$$
\begin{aligned}
& \llbracket J^{n} \boldsymbol{\sigma}\left(\boldsymbol{u}^{n}, p^{n}\right)\left(\boldsymbol{F}^{n}\right)^{-\mathrm{T}} \boldsymbol{n} \rrbracket+\frac{\rho^{\mathrm{S}} \epsilon}{\tau} \boldsymbol{u}^{n}=\frac{\rho^{\mathrm{S}} \epsilon}{\tau}\left(\dot{\boldsymbol{d}}^{n-1}+\tau \partial_{\tau} \dot{\boldsymbol{d}}^{n-1}\right) \\
& +\llbracket J^{n-1} \boldsymbol{\sigma}\left(\boldsymbol{u}^{n-1}, p^{n-1}\right)\left(\boldsymbol{F}^{n-1}\right)^{-\mathrm{T}} \boldsymbol{n} \rrbracket \quad \text { on } \quad \Sigma .
\end{aligned}
$$

3. Solid step: $\boldsymbol{d}^{n}: \Sigma \rightarrow \mathbb{R}^{3}, \dot{\boldsymbol{d}}^{n}: \Sigma \rightarrow \mathbb{R}^{3}$ and $\boldsymbol{a}^{n}: \Sigma \rightarrow \mathbb{R}^{3}$ with $\left|\boldsymbol{a}^{n}\right|=1$, satisfying (12) and

$$
\boldsymbol{f}^{\mathrm{f}, n}=-\llbracket J^{n} \boldsymbol{\sigma}\left(\boldsymbol{u}^{n}, p^{n}\right)\left(\boldsymbol{F}^{n}\right)^{-\mathrm{T}} \boldsymbol{n} \rrbracket \quad \text { on } \quad \Sigma .
$$

\section{Algorithm 6 Fully decoupled scheme.}

For $n \geq 2$ :

1. Update the fluid domain via (13);

2. Fluid viscous substep: find $\boldsymbol{u}^{n}: \Omega^{\mathrm{f}} \rightarrow \mathbb{R}^{3}$ satisfying (9) and

$$
\begin{aligned}
\llbracket J^{n} \boldsymbol{\sigma}\left(\boldsymbol{u}^{n}, p^{n-1}\right)\left(\boldsymbol{F}^{n}\right)^{-\mathrm{T}} \boldsymbol{n} \rrbracket+\frac{\rho^{\mathrm{s}} \epsilon}{\tau} \boldsymbol{u}^{n}=\frac{\rho^{\mathrm{s}} \epsilon}{\tau}\left(\dot{\boldsymbol{d}}^{n-1}+\tau \partial_{\tau} \dot{\boldsymbol{d}}_{\|}^{n-1}\right) & \\
+2 \mu\left(J \varepsilon(\boldsymbol{u}) \boldsymbol{F}^{-\mathrm{T}} \boldsymbol{n}\right)_{\|}^{n-1} & \text { on } \quad \Sigma .
\end{aligned}
$$

3. Fluid projection substep: find $\phi^{n}: \Omega^{\mathrm{f}} \rightarrow \mathbb{R}$ satisfying 10 and

$$
\frac{\tau}{\rho^{\mathrm{f}}} \frac{\partial \phi^{n}}{\partial \boldsymbol{n}}+\frac{\tau}{\rho^{\mathrm{s}} \epsilon} \llbracket \phi^{n} \rrbracket=\frac{\tau}{\rho^{\mathrm{s}} \epsilon} \llbracket \phi^{n-1} \rrbracket+\left(\boldsymbol{u}^{n-1}-\dot{\boldsymbol{d}}^{n-1}\right) \cdot \boldsymbol{n} \quad \text { on } \quad \Sigma .
$$

4. Solid step: find $\boldsymbol{d}^{n}: \Sigma \rightarrow \mathbb{R}^{3}, \dot{\boldsymbol{d}}^{n}: \Sigma \rightarrow \mathbb{R}^{3}$ and $\boldsymbol{a}^{n}: \Sigma \rightarrow \mathbb{R}^{3}$ with $\left|\boldsymbol{a}^{n}\right|=1$, satisfying (12) and

$$
\boldsymbol{f}^{\mathrm{f}, n}=-\llbracket J^{n} \boldsymbol{\sigma}\left(\boldsymbol{u}^{n}, p^{n}\right)\left(\boldsymbol{F}^{n}\right)^{-\mathrm{T}} \boldsymbol{n} \rrbracket \quad \text { on } \quad \Sigma .
$$

\subsection{Space discretization}

In Phase I, we take advantage of the symmetry of the problem along the $z$-axis to reduce the computational domain to half of the physical domain (see Figure 5). A symmetry boundary condition is enforced on the symmetry plane $\Gamma_{\text {sym }} \stackrel{\text { def }}{=}\{x=0\}$ :

$$
\boldsymbol{u} \cdot \boldsymbol{n}=0, \quad(\boldsymbol{\sigma}(\boldsymbol{u}, p) \boldsymbol{n})_{\|}=\mathbf{0} \quad \text { on } \quad \Gamma_{\mathrm{sym}} .
$$

The entire physical domain is considered as computational domain in Phase II.

The fluid domain is discretized with a tetrahedral mesh $\mathcal{T}_{h}$ (see Figures 5 and 6 , whereas a hexahedral $\mathcal{H}_{h}$ or quadrilateral mesh $\mathcal{Q}_{h}$ is considered for the solid domain, depending on wether it is a volume (Figures 5(a) and 6(a)) or a surface (Figures 5(b) and 6(b)). The meshes are built 
in such a way that the nodes lying on the interface are matching (see 43]). The current fluid mesh $\mathcal{T}_{h}^{n}$ of $\Omega^{\mathrm{f}, n}$ is obtained by displacing the nodes of $\mathcal{T}_{h}$ by $\boldsymbol{d}_{h}^{\mathrm{f}, n}$ (see Section 3.3 ).

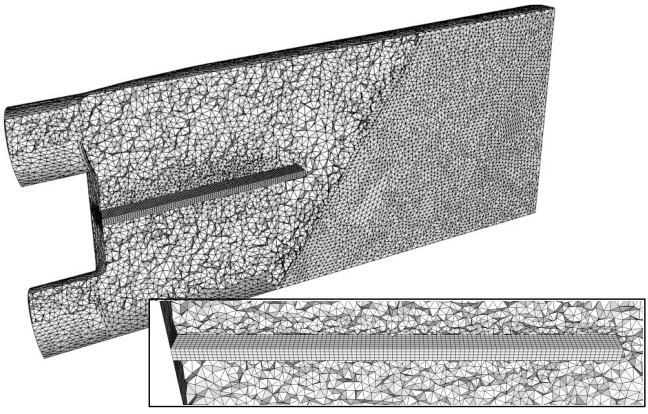

(a) $\mathcal{T}_{h}: 485331$ tetrahedra, $\mathcal{H}_{h}: 4000$ hexahedra.

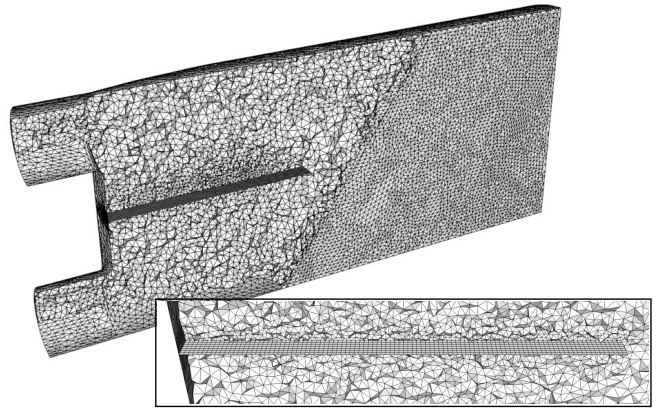

(b) $\mathcal{T}_{h}: 476810$ tetrahedra, $\mathcal{Q}_{h}: 1000$ quadrilaterals.

Figure 5: Meshes for Phase I: (a) with 3D solid and (b) with shell solid.

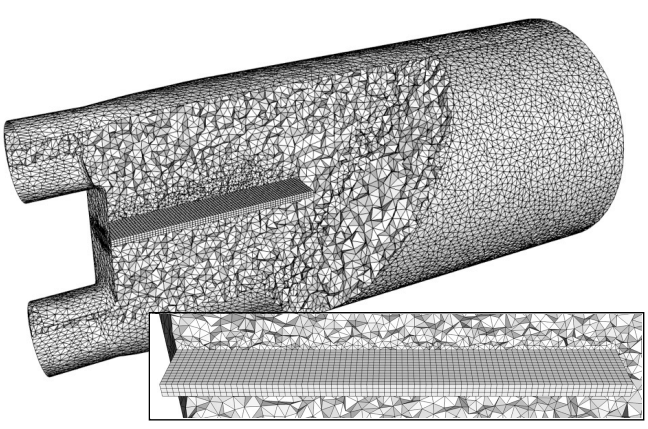

(a) $\mathcal{T}_{h}: 624440$ tetrahedra, $\mathcal{H}_{h}: 3840$ hexahedra.

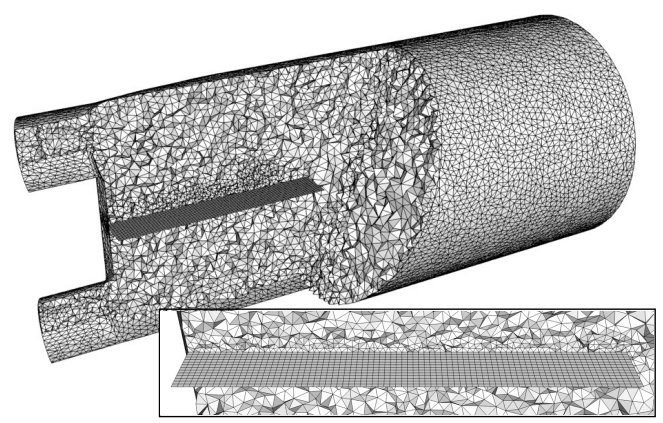

(b) $\mathcal{T}_{h}: 609752$ tetrahedra, $\mathcal{Q}_{h}: 1280$ quadrilaterals.

Figure 6: Meshes for Phase II: (a) with 3D solid and (b) with shell solid.

The fluid equations are discretized in space using $\mathbb{P}_{1}$ finite elements. As usual, we use the subscript $h$ to denote the discrete approximations. For Algorithms 1, 2 and 5 , we resort to the SUPG/PSPG method to stabilize the velocity/pressure pair and the convective effects (see, e.g., [76). Furthermore, a backflow stabilization method is used on the outlet boundary (see, e.g., $[58,14])$. In summary, we add the following stabilization terms to the fully discrete fluid problem:

$$
\begin{aligned}
\sum_{K \in \mathcal{T}_{h}^{n}} \int_{K} \tau_{K}\left(\rho^{\mathrm{f}}\left(\boldsymbol{u}_{h}^{n-1} \cdot \nabla\right) \boldsymbol{u}_{h}^{n}+\nabla p_{h}^{n}-\rho^{\mathrm{f}} \boldsymbol{g}\right) & \cdot\left(\rho^{\mathrm{f}}\left(\boldsymbol{u}_{h}^{n-1} \cdot \nabla\right) \boldsymbol{v}_{h}+\nabla q_{h}\right) \\
& +\frac{\rho^{\mathrm{f}}}{2} \int_{\Gamma_{\text {out }}}\left(\boldsymbol{u}_{h}^{n} \cdot \boldsymbol{n}\right)_{-} \boldsymbol{u}_{h}^{n} \cdot \boldsymbol{v}_{h},
\end{aligned}
$$

where $\boldsymbol{v}_{h}$ and $q_{h}$ are the discrete velocity and pressure test functions, the symbol $\left(\boldsymbol{u}^{n} \cdot \boldsymbol{n}\right)_{-}$ denotes the negative part of $\boldsymbol{u}^{n} \cdot \boldsymbol{n}$ and $\tau_{K}$ is the SUPG/PSPG stabilization parameter. For Algorithms 3 , 4 and 6. we split the stabilization $(19)$ in its velocity and pressure parts, adding 
the following SUPG contribution to the convective-viscous substep (9):

$$
\sum_{K \in \mathcal{T}_{h}^{n}} \int_{K} \tau_{K}\left(\rho^{\mathrm{f}}\left(\boldsymbol{u}_{h}^{n-1} \cdot \nabla\right) \boldsymbol{u}_{h}^{n}+\nabla p_{h}^{n-1}-\rho^{\mathrm{f}} \boldsymbol{g}\right) \cdot\left(\rho^{\mathrm{f}}\left(\boldsymbol{u}_{h}^{n-1} \cdot \nabla\right) \boldsymbol{v}_{h}\right)+\frac{\rho^{\mathrm{f}}}{2} \int_{\Gamma_{\text {out }}}\left(\boldsymbol{u}_{h}^{n} \cdot \boldsymbol{n}\right)_{-} \boldsymbol{u}_{h}^{n} \cdot \boldsymbol{v}_{h},
$$

and the following PSPG contribution to the projection step (4):

$$
\sum_{K \in \mathcal{T}_{h}^{n}} \int_{K} \tau_{K}\left(\rho^{\mathrm{f}}\left(\boldsymbol{u}_{h}^{n-1} \cdot \nabla\right) \boldsymbol{u}_{h}^{n}+\nabla p_{h}^{n}-\rho^{\mathrm{f}} \boldsymbol{g}\right) \cdot \nabla q_{h} .
$$

In Algorithms 2 and 4 6, which involve the thin-walled solid model (6), discontinuous approximations of the pressure are included by duplicating the interface pressure degrees of freedom.

The spatial discretization of the solid elastodynamics equations in Algorithms 1 and 3 is performed with $\mathbb{Q}_{1}$ finite elements. The shell equation in Algorithms 2 and 46 , is discretized in space by quadrilateral MITC4 elements (see [21, Section 8.2.1]), with 5 degrees of freedom per node in the increments.

Remark 3.1. In order to guarantee a correct energy balance of the fully discrete approximations across the interface, all the interface fluid stresses involved in the dynamic coupling conditions (3) 3 and (7) 3 are evaluated in a weak sense as varational residuals (see, e.g., [50, [57, 80]). The sole exception are the projection based semi-implicit coupling schemes of Section 3.1.2. for which pressure stresses on the interface are evaluated directly as face-wise integrals. This avoids the computation of an intermediate fluid velocity, necessary for the evaluation of the residual. Numerical evidence (see, e.g., [32]) indicates that this simplification does not compromise either stability or accuracy.

\subsection{Mesh update technique}

We adopt a mesh moving technique which builds on the equations of linear elasticity. The associated pseudo Lamé coefficients are updated element-wise to stiffen the smallest elements (see 71]), whose deformation can potentially make the mesh invalid. Moreover, in order to enhance robustness, the lifted displacement is computed incrementally, using the previous configuration of the mesh. More precisely, given $\left.\boldsymbol{d}_{h}^{n-1}\right|_{\Sigma}$, we compute the lifted displacement $\boldsymbol{d}_{h}^{\mathrm{f}, n}=\mathcal{L}\left(\left.\boldsymbol{d}_{h}^{n-1}\right|_{\Sigma}\right)$

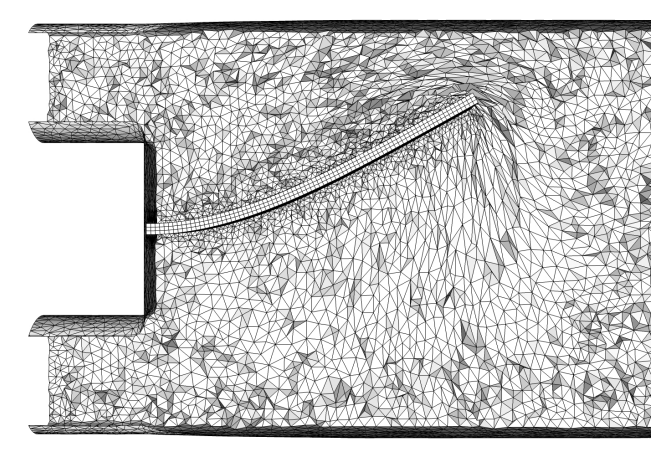

(a) Horizontal perspective.

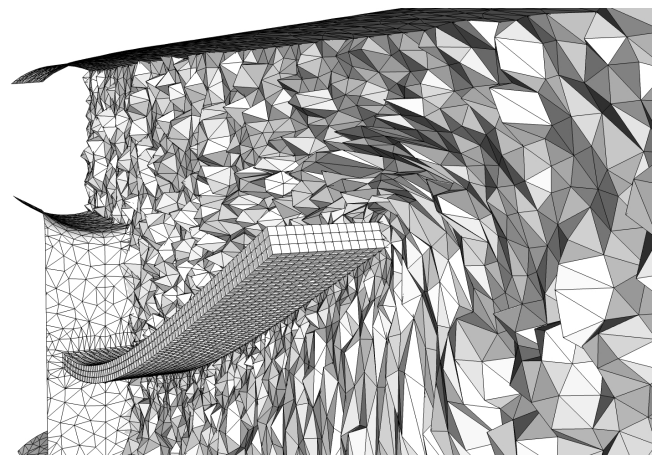

(b) Detail of the deformed mesh.

Figure 7: Initial condition for the meshes. Application of the operator $\mathcal{L}(\cdot)$.

by the following procedure: 
1. Compute the solid displacement increment at the reference interface:

$$
\delta \boldsymbol{d}_{h}^{n-1}=\left.\boldsymbol{d}_{h}^{n-1}\right|_{\Sigma}-\left.\boldsymbol{d}_{h}^{n-2}\right|_{\Sigma}
$$

2. Compute the lifting $\delta \boldsymbol{d}_{h}^{\mathrm{f}, n}$ of the increment $\delta \boldsymbol{d}_{h}^{n-1}$ solving the following linear elasticity problem in the previous configuration $\Omega^{\mathrm{f}, n-1}$ :

$$
\left\{\begin{array}{r}
\left.\delta \boldsymbol{d}_{h}^{\mathrm{f}, n}\right|_{\Sigma^{n-1}}=\delta \boldsymbol{d}_{h}^{n-1} \circ\left(\mathcal{A}_{h}^{n-1}\right)^{-1},\left.\quad \delta \boldsymbol{d}_{h}^{\mathrm{f}, n}\right|_{\Omega^{\mathrm{f}, n-1} / \Sigma^{n-1}}=\mathbf{0} \\
\sum_{K \in \mathcal{T}_{h}^{n-1}} \int_{K}\left(L_{1, K} \operatorname{div}\left(\delta \boldsymbol{d}_{h}^{\mathrm{f}, n}\right) \operatorname{div} \boldsymbol{w}_{h}+2 L_{2, K} \boldsymbol{\varepsilon}\left(\delta \boldsymbol{d}_{h}^{\mathrm{f}, n}\right): \boldsymbol{\varepsilon}\left(\boldsymbol{w}_{h}\right)\right)=0 \quad \forall \boldsymbol{w}_{h}
\end{array}\right.
$$

where the Lamé parameters $L_{1, K}, L_{2, K}$ are defined element-wise as

$$
L_{1, K}=\frac{\widetilde{L}_{1}}{V_{K}}, \quad L_{2, K}=\frac{\widetilde{L}_{2}}{V_{K}},
$$

with $\widetilde{L}_{1}, \widetilde{L}_{2}>0$ given constants and $V_{K}$ denotes the volume of the element $K \in \mathcal{T}_{h}^{n-1}$.

3. Update the fluid displacement in the reference configuration:

$$
\boldsymbol{d}_{h}^{\mathrm{f}, n}=\boldsymbol{d}_{h}^{\mathrm{f}, n-1}+\delta \boldsymbol{d}_{h}^{\mathrm{f}, n} \circ \mathcal{A}_{h}^{n-1}
$$

An example of a deformed mesh, involving large interface deflections, obtained through this procedure is displayed in Figure 7 .

\section{Numerical results and comparison with experimental data}

The numerical results presented below were generated with a partitioned master/slave implementation of Algorithms 1 6. Data exchanges across the fluid-solid interface are managed by a master code (based on PVM [41), with the slaves being the fluid and solid solvers (see, e.g., 42, 53 ). Different parallel methods are used for the solid and the fluid. The parallel structural solver uses Newton iterations at each time-step. The resulting tangent systems are solved with a balancing domain decomposition method (see, e.g., [56, 51, 49]). The fluid solver FELiScE (see [28]) is based on PETSc (see [10, 11, 12]) and uses an additive Schwarz algorithm (see, e.g., 70]) with local ILU preconditioning.

\subsection{Initial configuration}

According to (5), the Phase I and II experiments are initialized with the solid configuration in its hydrostatic equilibrium $\boldsymbol{d}_{0}$. An approximation of this configuration is used to initialize $\boldsymbol{d}^{0}$ in Algorithms 1, 6. This approximation can be obtained as the steady state solution provided by Algorithms 16 with $\boldsymbol{d}^{0}=\mathbf{0}, \boldsymbol{u}_{\text {top }}^{n}=\boldsymbol{u}_{\text {bot }}^{n}=\mathbf{0}$. Figure 8 reports the solid hydrostatic equilibrium obtained with the implicit schemes (Algorithms 1 2]. Note that the thin-walled shell model (Algorithm 2) provides slightly larger deflections in both phases. Similar results are obtained with the rest of the methods.

It should be noted that these numerical approximations underestimate the maximal deflections measured in the experiment for Phases I and II at rest, which are $29.5 \mathrm{~mm}$ and $25.65 \mathrm{~mm}$, respectively. In particular, such a difference is not observed between the results for Phases I and II in Figure 8, despite the modifications introduced in the fluid physical parameters (see Sections 2.3 .12 .3 .2 .

$\mathrm{RR} \mathrm{n}^{\circ} \mathrm{RR}-8824$ 


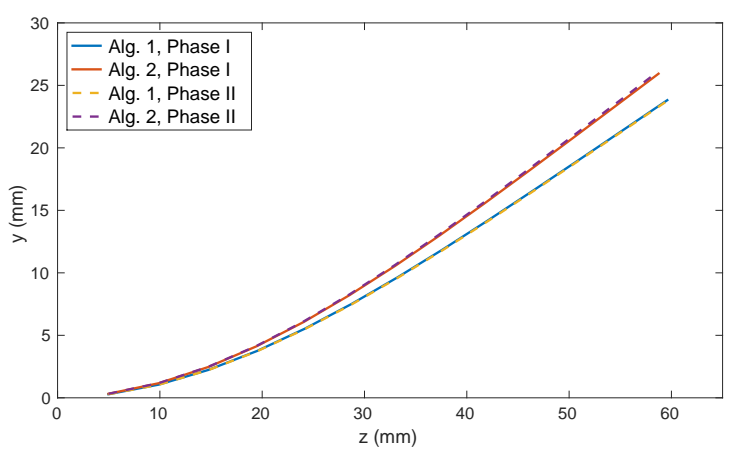

Figure 8: Initial solid configuration (center line) for Phase I and Phase II obtained with Algorithms 1 2. The axis labels indicate the $y$ - and $z$-coordinates in the referential of Figure 1 .

\subsection{Phase I experiment}

The solid filament is initially at the hydrostatic equilibrium computed in Section 4.1. The constant flow rate described in Section 2.3.1 is imposed on the top and bottom inlet boundaries. We have simulated 6000 time-steps of constant length $\tau=2.5 \cdot 10^{-3} s$ with Algorithms 16 . After a brief transition phase, the system reaches a steady state equilibrium with the filament deflected and holding a stable position. Figure 9 shows the steady state velocity field magnitude and the deformed solid configuration provided by Algorithm 1 (3D solid model) and Algorithm 2 (shell solid model). Similar results are obtained with the rest of methods, that are omitted here for the sake of conciseness.

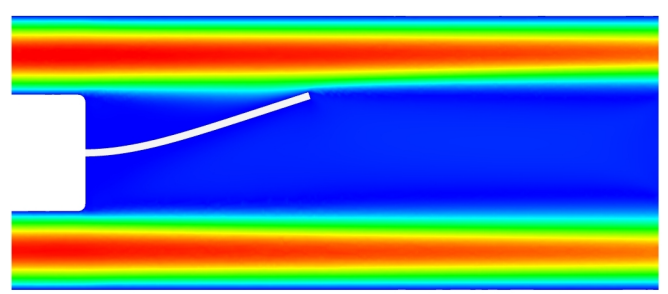

(a) 3D solid model (Algorithm 1).

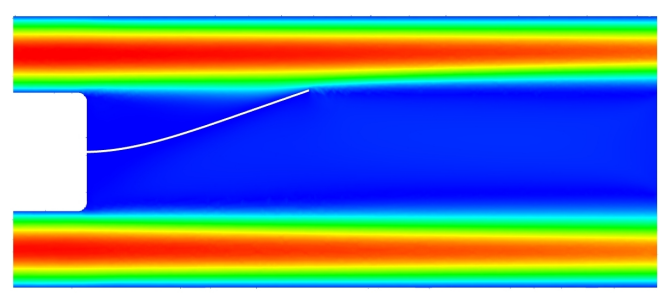

(b) Shell solid model (Algorithm 2p.

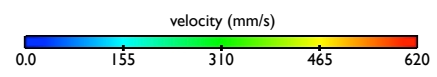

Figure 9: Steady state velocity magnitude and deformed solid configuration in Phase I.

In Figure 10, we compare the measured final filament configuration and the approximations provided by Algorithms 1 6 . In order to better highlight the differences, the range of the $y$-coordinate has been rescaled with respect to Figure 8. We can clearly see that all the methods considered provide numerical solutions that are very close to the experimental data. Moreover, the results obtained with Algorithms 1 and 3 (3D solid model) are practically the same. Similar behaviour is observed for Algorithms 2 and 4 6 (shell solid model). This is consistent with the fact that, for steady state solutions, the choice of the coupling scheme has no impact on the quality of the approximation, which is only driven by the solid modelling assumptions and the approximation in space. Figure 10 indicates that the 3D solid model gives slightly better results than the shell model, which tends to overestimate the deflection of the filament. 


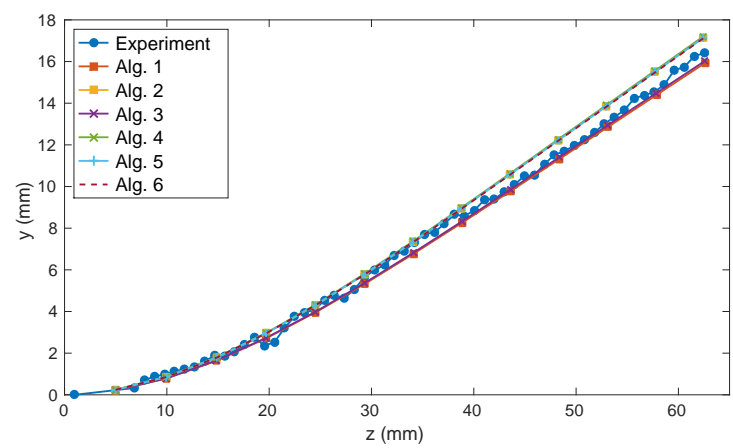

Figure 10: Deformed solid configuration (center line) at steady state in Phase I. Comparison of the experimental data with the predictions obtained with Algorithms 1 6. The axis labels indicate the $y$ - and $z$-coordinates in the referential of Figure 1 .

Experimental measurements of the fluid velocity components are available at the cutting planes $z=10$ and $z=30$. In Figure 11 , we compare the experimental results with the numerical approximations obtained with Algorithm 1 (monolithic fluid solver and 3D solid model) and Algorithm 6 (incremental pressure-correction fluid solver and shell solid model). Note that the numerical results are presented with the spatial resolution of the experimental data. As above, very similar results are obtained with the rest of algorithms. Figure 11 shows a good agreement between the simulations and the experimental data, irrespectively of the $3 \mathrm{D}$ or shell nature of the solid model.

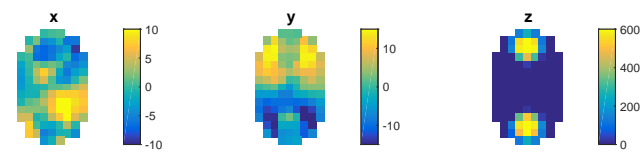

(a) Experiment on $z=10$.
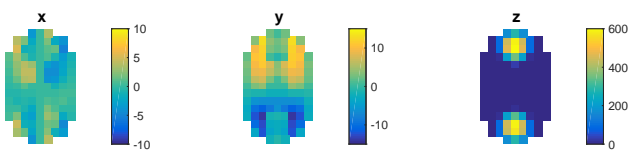

(c) Algorithm 1 on $z=10$.
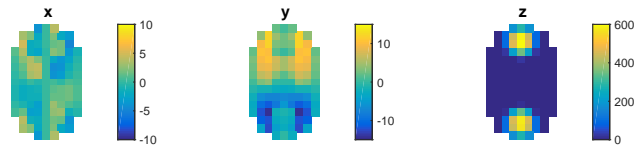

(e) Algorithm 6 on $z=10$.
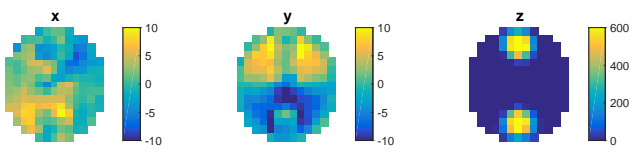

(b) Experiment on $z=30$.
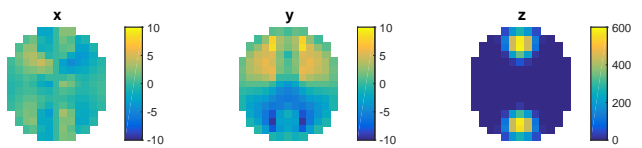

(d) Algorithm 1 on $z=30$.
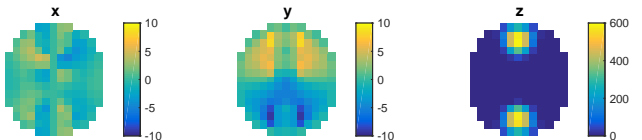

(f) Algorithm 6 on $z=30$.

Figure 11: Fluid velocity components at steady state in Phase I. Comparison of the experimental data with the predictions obtained with Algorithms 1 and 6 . The numerical results are presented with the same spatial resolution as the experimental data. Units are in $\mathrm{mm} / \mathrm{s}$.

In order to provide some insight into the computational performance of the different numerical methods, we have reported in Table 4 a comparison of the elapsed CPU-times (dimensionless) obtained with Algorithms 116 during the first 10 time-steps. The experiments were run in the 
same machine, with 16 and 8 processors allocated for the fluid and the solid solvers, respectively. The semi-implicit schemes (Algorithms 34 are around 6 and 8 times faster than the strongly coupled schemes (Algorithms 1 2). This CPU-time saving is due to the reduced computational complexity of Step 3 in Algorithms 34 with respect to Step 2 of Algorithms 1,2 . Note that a slight speed-up is obtained when the shell model (Algorithms 2 and 4 ) is used instead of the 3D solid model (Algorithms 1 and 3). This is consistent with the fact that, for the considered spatial discretizations, the shell model yields a discrete problem with a lower number of degrees of freedom (see Section 3.2). The best computational performance is obtained with the explicit

\begin{tabular}{|c|c|c|c|c|c|}
\hline Alg. 1 & Alg. $[2$ & Alg. 3 & Alg. 4 & Alg. 5 & Alg. 6 \\
\hline 18 & 17 & 3 & 2 & 2 & 1 \\
\hline
\end{tabular}

Table 4: Elapsed CPU-time (dimensionless) for Algorithms 1, 6 in Phase I.

coupling schemes, Algortihms 5 6, which invoke the fluid and solid solvers only once per timestep. Note that the speed-up of Algorithm 6 with respect to Algortihms 5 is exclusively due to the fractional-step time-marching in the fluid.

\subsection{Phase II experiment}

The solid filament is initially in its hydrostatic equilibrium (see Section 4.1). On the inlet boundaries, we impose the pulsatile flow profile described in Section 2.3.2. We have simulated 6000 time-steps of constant length $\tau=10^{-3} s$ with Algorithms 1 , 6 , which corresponds to a full cycle $(6 s)$ of the pulsatile flow.

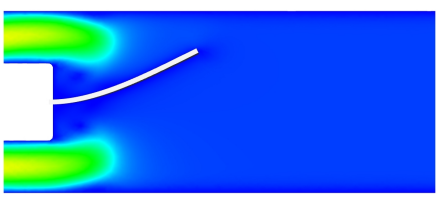

(a) $t=0.721 \mathrm{~s}$

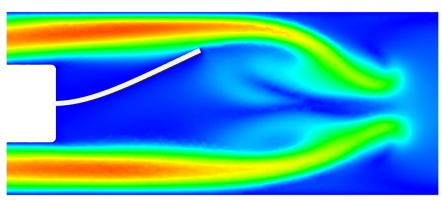

(d) $t=2.017 \mathrm{~s}$

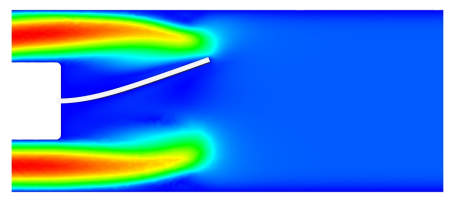

(b) $t=1.153 \mathrm{~s}$

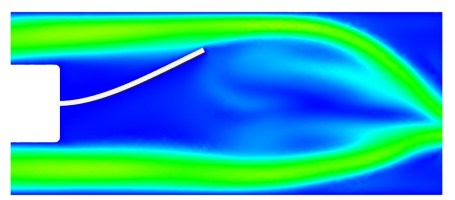

(e) $t=2.449 \mathrm{~s}$

velocity $(\mathrm{mm} / \mathrm{s})$

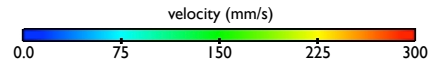

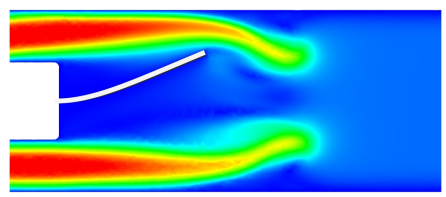

(c) $t=1.585 \mathrm{~s}$

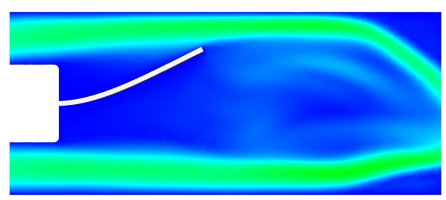

(f) $t=2.881 \mathrm{~s}$

Figure 12: Snapshots of the fluid velocity magnitude and deformed solid configuration in Phase II with Algorithm 1 .

Figures 12 and 14 present some snapshots of the fluid velocity magnitude at different time instants obtained with Algorithm 1 (3D solid model) and Algorithm 2 (shell solid model), respectively. The fluid front hits the filament, and makes it to deflect. As soon as the fluid flow diminishes, the filament returns to its original position. For illustration purposes, Figures 13 and 15 report some vortical structures obtained as iso-surfaces of the $Q$-criterion. The rest of the 


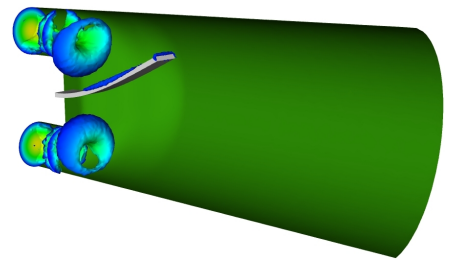

(a) $t=0.721 \mathrm{~s}$

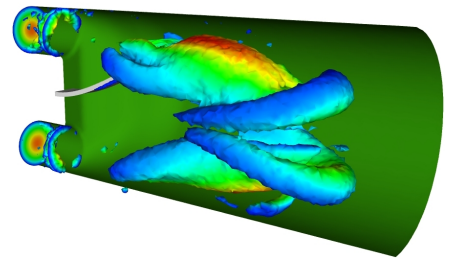

(d) $t=2.017 \mathrm{~s}$

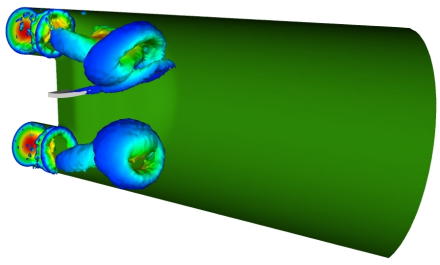

(b) $t=1.153 \mathrm{~s}$

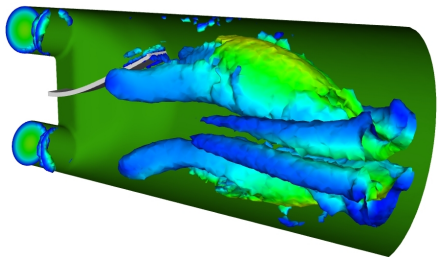

(e) $t=2.449 \mathrm{~s}$

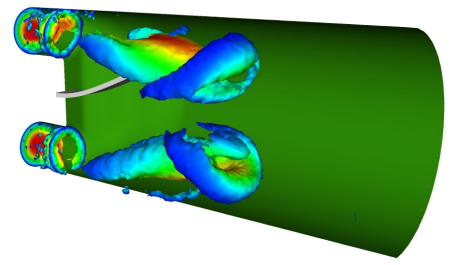

(c) $t=1.585 \mathrm{~s}$

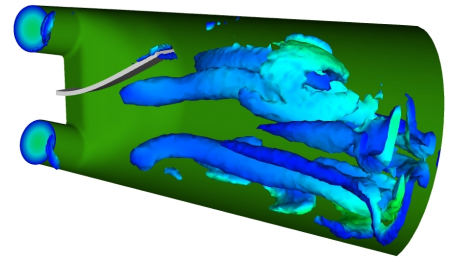

(f) $t=2.881 \mathrm{~s}$

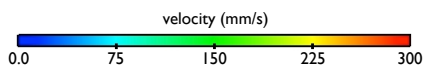

Figure 13: Isosurface $Q=12$ of the $Q$-criterion colored by velocity. (Algorithm 1 in Phase II.

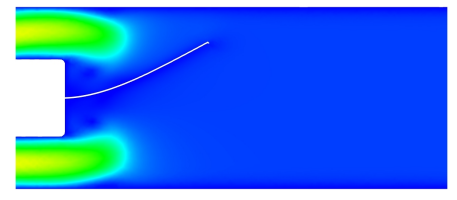

(a) $t=0.721 \mathrm{~s}$

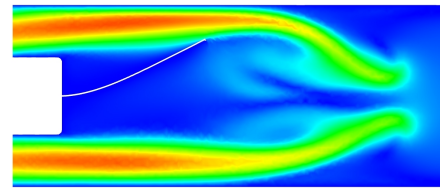

(d) $t=2.017 \mathrm{~s}$

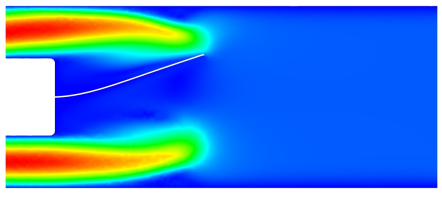

(b) $t=1.153 \mathrm{~s}$

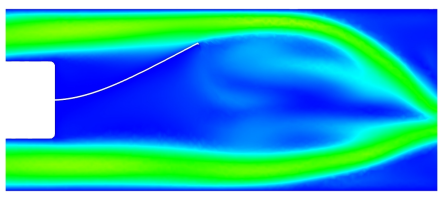

(e) $t=2.449 \mathrm{~s}$

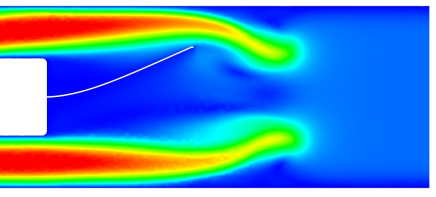

(c) $t=1.585 \mathrm{~s}$

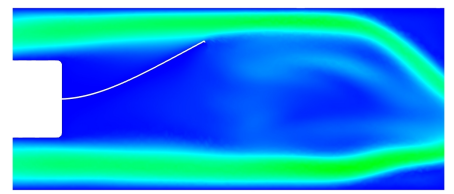

(f) $t=2.881 \mathrm{~s}$

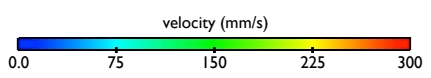

Figure 14: Snapshots of the fluid velocity magnitude and deformed solid configuration in Phase II with Algorithm 2

algorithms deliver very similar results. We can clearly see in these figures the initial deflection of the structure due to the impact of the fluid jet.

Figure 16 presents a comparison of the measured deflections and the results obtained with Algorithms 1 6 at different time-steps in $[0,3]$ (where the main transient phenomena occur). Note that the experimentally measured deflection is truncated over $x \in[0,53.193]$, whereas the whole results are displayed for the simulations. We can observe that all the numerical methods are able to capture the main dynamics of the system. In order to provide further insight into this comparison, Figure 17 displays the $y$-component of the point in the structure with coordinate 


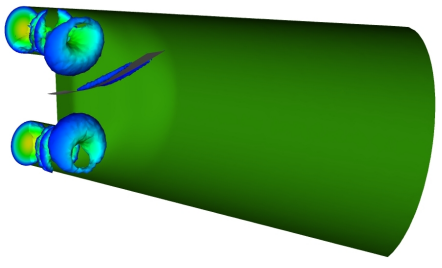

(a) $t=0.721 \mathrm{~s}$

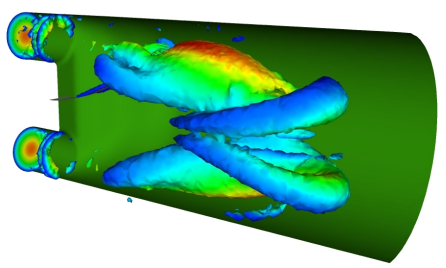

(d) $t=2.017 \mathrm{~s}$

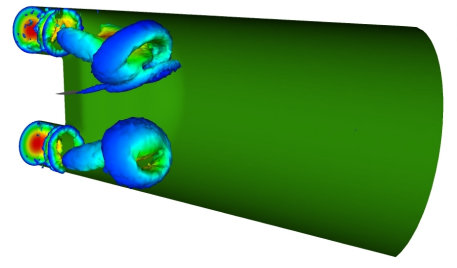

(b) $t=1.153 \mathrm{~s}$

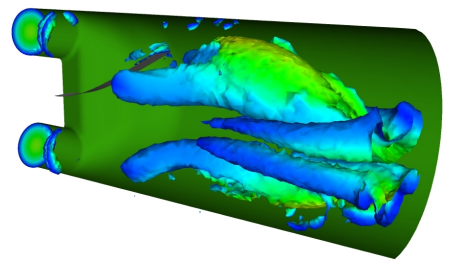

(e) $t=2.449 \mathrm{~s}$

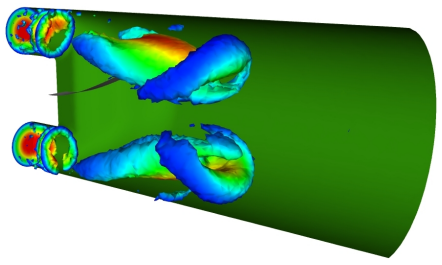

(c) $t=1.585 \mathrm{~s}$

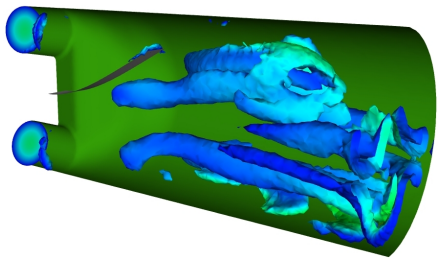

(f) $t=2.881 \mathrm{~s}$

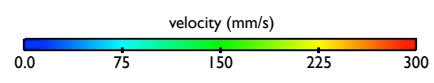

Figure 15: Isosurface $Q=12$ of the $Q$-criterion colored by velocity magnitude in Phase II with Algorithm 2 .

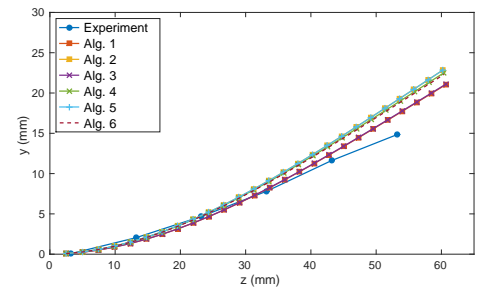

(a) $t=0.721 \mathrm{~s}$

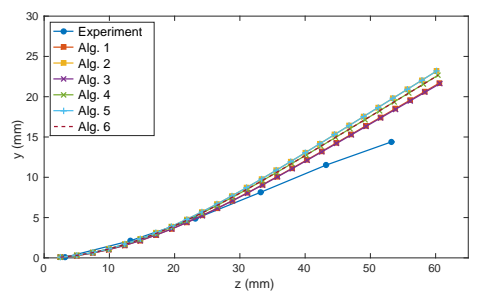

(d) $t=2.017 \mathrm{~s}$

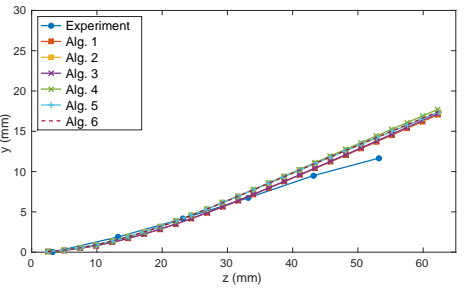

(b) $t=1.153 \mathrm{~s}$

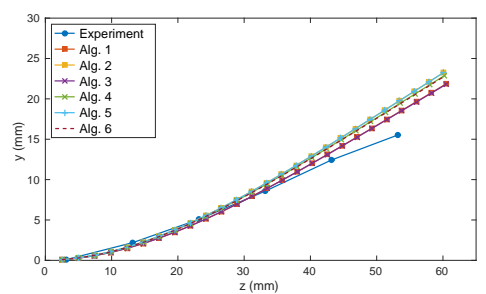

(e) $t=2.449 \mathrm{~s}$

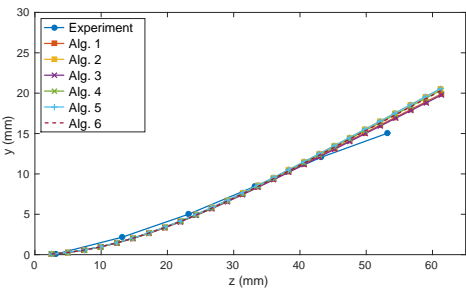

(c) $t=1.585 \mathrm{~s}$

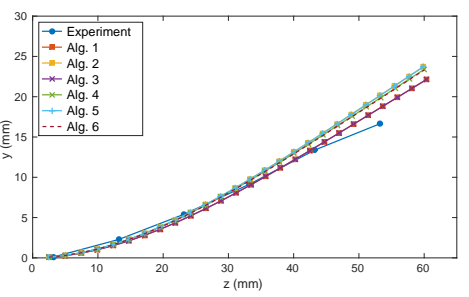

(f) $t=2.881 \mathrm{~s}$

Figure 16: Deflection of the silicone filament in Phase II at different time instants during the window $[0,3] s$. Comparison of the experimental data with the predictions obtained with Algorithms 16 .

$x=53.193$ for all $t \in[0,6]$. We can see that the first impact of the fluid into the solid causes the main deflection of the filament at $t=1.153 \mathrm{~s}$. The instant in which the filament reaches this maximum deflection is perfectly predicted by all the methods (see also Figure 16(b)). Afterwards, the solid starts to go up due to the buoyancy force and hits again the fluid jet at $t=1.585 \mathrm{~s}$. 
Then, a second, much more mitigated, bounce starts which is, however, captured with a delay in all the simulations. Figure 17 shows also the general tendency of the shell model to overestimate the deflection of the filament, especially during the second half of the cycle.

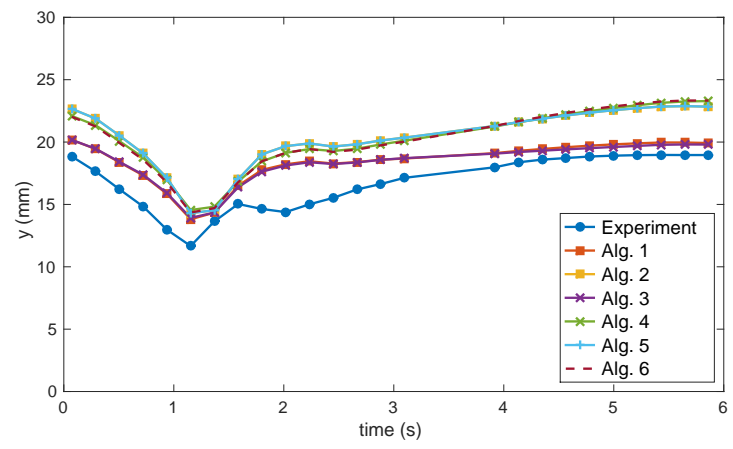

Figure 17: Track of $y$-component of the point in the structure with coordinate $x=53.193$ for all $t \in[0,6]$ in Phase II.

Experimental measurements of the fluid velocity components at different time-steps were obtained at the cutting planes $z=3.5, z=13.5, z=23.5$ and $z=33.5$. Figure 18 shows the experimental results and the numerical approximations obtained, at two different time-steps, with Algorithm 1] (monolithic fluid solver and 3D solid model) and Algorithm 6 (incremental pressure-correction fluid solver and shell solid model) at $z=33.5$ (the furthest from the inlet boundary, which corresponds to the most difficult scenario). Note that the numerical results are presented with the spatial resolution of the experimental data. Although slightly diffusive, the simulations are in good agreement with the experimental data (irrespectively of the fluidsolver/solid-model used). In particular, the main patterns of the flow are clearly captured. Similar results, not reported here for the sake of conciseness, were obtained with the rest of methods and for the rest of scanned planes.

Finally, in Table 5, we compare the performance of Algorithms 1 6 in terms of the elapsed CPU-time during the first 10 time-steps. This is carried out in the same machine, with 16 and 8 processors allocated for the fluid and the solid solvers, respectively. As in the simulations of Phase I, superior performance is obtained with the loosely coupled and semi-implicit methods.

\begin{tabular}{|c|c|c|c|c|c|}
\hline Alg. 1 & Alg. 2 & Alg. 3 & Alg. 4 & Alg. 5 & Alg. 6 \\
\hline 20 & 18 & 2.5 & 2.5 & 2 & 1 \\
\hline
\end{tabular}

Table 5: Elapsed CPU-time (dimensionless) for Algorithms 1 6 in Phase II.

It is worth noting that the elapsed CPU-times for Algorithms 14 depend on the type of solvers and preconditioners used for the solution of the coupled problems involved in Step 2 of Algorithms 1, 2 and Step 3 of Algorithms 3.4. Here, as stated in Sections 3.1.1 3.1.2, these coupled problems are solved with a (parameter free) partitioned solution procedure based on interface Newton-GMRES Dirichlet-Neumann iterations. Nevertheless, the global ranking of Tables 45 is independent of this choice, since the efficiency of the coupling schemes is dictated by the computational complexity of the associated coupled problem. 

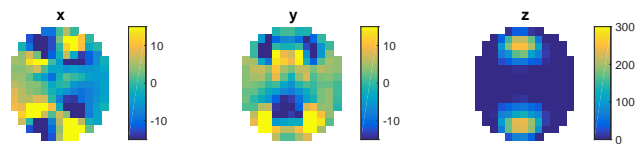

(a) Experiment at $t=1.153$.
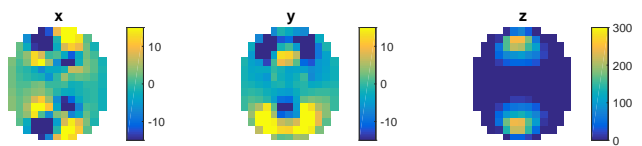

(c) Algorithm 1 at $t=1.153$.
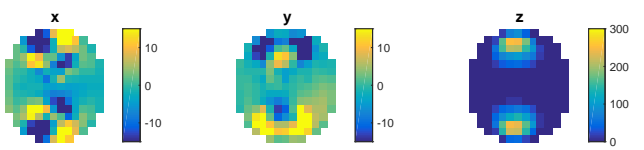

(e) Algorithm 6 at $t=1.153$.
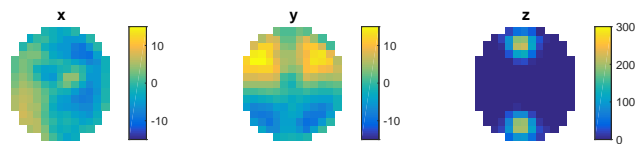

(b) Experiment at $t=2.449$.
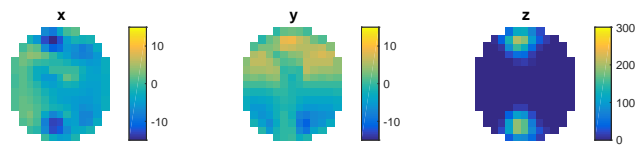

(d) Algorithm 1 at $t=2.449$.
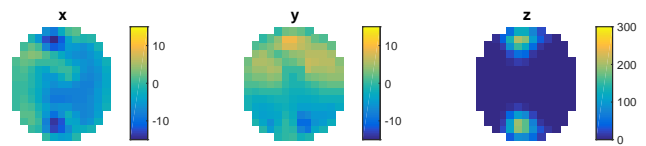

(f) Algorithm 6 at $t=2.449$.

Figure 18: Fluid velocity components in Phase II. Comparison of the experimental data with the predictions obtained with Algorithms 1 and 6 on the cutting plane $z=33.5$. The numerical results are presented with the same spatial resolution as the experimental data. Units are in $\mathrm{mm} / \mathrm{s}$.

\section{Conclusion}

Different partitioned solution procedures have been compared and validated using the experimental results of the FSI benchmark presented in [39. All the methods discussed are algorithmically parameter free and represent an important sample of the state-of-the-art in coupling schemes for FSI.

The comparisons in Section 4 showed, in general, a very good agreement between the numerical and the experimental results, particularly, in Phase I where the matching is excellent. A slight deviation is observed in the case of the coupling with the shell model, which can be related to the approximation of the Archimedes force.

With regard to the computational efficiency, the comparisons indicate that the best performance is obtained with the splitting schemes of Algorithms 3 and 6 , respectively, in the case of the coupling with a $3 \mathrm{D}$ and a shell solid model. It should be noted also that these results confirm that strong coupling (Algorithms 1 2 can be avoided via semi-implicit or explicit coupling (Algorithms 36 without compromising both stability and accuracy, and at significantly reduced computational cost (see, e.g., Table 5).

Further investigations or improvements of the present work can explore various directions. From the modeling point of view, we could consider the coupling with three-dimensional shell models (see, e.g., [21]), which would bypass the introduction of an ad hoc Archimedes surface force. From the numerical point of view, it would be worth investigating to what extend the numerical results of Phase II can be improved by using further refined discretizations and/or second-order time-discretizations. With regard to the latter point, preliminary results (not reported here) show no significant differences with a second-order time-stepping in the solid. Another interesting aspect, not addressed in the present work, is the inclusion in the comparisons of alternative immersed FSI formulations based on unfitted meshes (see, e.g., [3, 47, 1]). 


\section{Acknowledgements}

This work was supported by the French National Research Agency (ANR) through the EXIFSI project (ANR-12-JS01-0004). We are grateful to our colleague Jean-Frédéric Gerbeau (Inria \& Sorbonne Universités) for all his support and fruitful discussions.

\section{References}

[1] F. Alauzet, B. Fabrèges, M. A. Fernández, and M. Landajuela. Nitsche-XFEM for the coupling of an incompressible fluid with immersed thin-walled structures. Comput. Methods Appl. Mech. Engrg., 301:300-335, 2016.

[2] M. Astorino, F. Chouly, and M. A. Fernández. Robin based semi-implicit coupling in fluidstructure interaction: Stability analysis and numerics. SIAM J. Sci. Comput., 31(6):40414065,2009 .

[3] M. Astorino, J.-F. Gerbeau, O. Pantz, and K.-F. Traoré. Fluid-structure interaction and multi-body contact: application to aortic valves. Comput. Methods Appl. Mech. Engrg., 198(45-46):3603-3612, 2009.

[4] M. Astorino and C. Grandmont. Convergence analysis of a projection semi-implicit coupling scheme for fluid-structure interaction problems. Numer. Math., 116:721-767, 2010.

[5] S. Badia, F. Nobile, and C. Vergara. Fluid-structure partitioned procedures based on Robin transmission conditions. J. Comp. Phys., 227:7027-7051, 2008.

[6] S. Badia, F. Nobile, and C. Vergara. Robin-Robin preconditioned Krylov methods for fluidstructure interaction problems. Comput. Methods Appl. Mech. Engrg., 198(33-36):27682784,2009 .

[7] S. Badia, A. Quaini, and A. Quarteroni. Modular vs. non-modular preconditioners for fluid-structure systems with large added-mass effect. Comput. Methods Appl. Mech. Engrg., 197(49-50):4216-4232, 2008.

[8] S. Badia, A. Quaini, and A. Quarteroni. Splitting methods based on algebraic factorization for fluid-structure interaction. SIAM J. Sci. Comput., 30(4):1778-1805, 2008.

[9] H. Baek and G.E. Karniadakis. A convergence study of a new partitioned fluid-structure interaction algorithm based on fictitious mass and damping. J. Comput. Phys., 231(2):629 $652,2012$.

[10] Satish Balay, Shrirang Abhyankar, Mark F. Adams, Jed Brown, Peter Brune, Kris Buschelman, Victor Eijkhout, William D. Gropp, Dinesh Kaushik, Matthew G. Knepley, Lois Curfman McInnes, Karl Rupp, Barry F. Smith, and Hong Zhang. PETSc Web page, 2014.

[11] Satish Balay, Shrirang Abhyankar, Mark F. Adams, Jed Brown, Peter Brune, Kris Buschelman, Victor Eijkhout, William D. Gropp, Dinesh Kaushik, Matthew G. Knepley, Lois Curfman McInnes, Karl Rupp, Barry F. Smith, and Hong Zhang. PETSc users manual. Technical Report ANL-95/11 - Revision 3.5, Argonne National Laboratory, 2014.

[12] Satish Balay, William D. Gropp, Lois Curfman McInnes, and Barry F. Smith. Efficient management of parallelism in object oriented numerical software libraries. In E. Arge, A. M. Bruaset, and H. P. Langtangen, editors, Modern Software Tools in Scientific Computing, pages 163-202. Birkhäuser Press, 1997. 
[13] J.W. Banks, W.D. Henshaw, and D.W. Schwendeman. An analysis of a new stable partitioned algorithm for FSI problems. Part II: Incompressible flow and structural shells. $J$. Comput. Phys., 268:399-416, 2014.

[14] Y Bazilevs, JR Gohean, TJR Hughes, RD Moser, and Y Zhang. Patient-specific isogeometric fluid-structure interaction analysis of thoracic aortic blood flow due to implantation of the jarvik 2000 left ventricular assist device. Computer Methods in Applied Mechanics and Engineering, 198(45):3534-3550, 2009.

[15] C. Bertoglio, D. Barber, N. Gaddum, V. Valverde, M. Rutten, P. Beerbaum, P. Moireau, R. Hose, and J.-F. Gerbeau. Identification of artery wall stiffness: In vitro validation and in vivo results of a data assimilation procedure applied to a $3 \mathrm{~d}$ fluidstructure interaction model. J. Biomech., 47(5):1027-1034, 2014.

[16] M. Bukac, C. Canic, R. Glowinski, T. Tambaca, and A. Quaini. Fluid-structure interaction in blood flow capturing non-zero longitudinal structure displacement. J. Comp. Phys., 235(0):515-541, 2013.

[17] M. Bukac, S. Canic, R. Glowinski, B. Muha, and A. Quaini. A modular, operator-splitting scheme for fluid-structure interaction problems with thick structures. Int. J. Numer. Meth. Fluids., 74(8):577-604, 2014.

[18] E. Burman and M. A. Fernández. Stabilization of explicit coupling in fluid-structure interaction involving fluid incompressibility. Comput. Methods Appl. Mech. Engrg., 198(5-8):766784,2009 .

[19] E. Burman and M. A. Fernández. Explicit strategies for incompressible fluid-structure interaction problems: Nitsche type mortaring versus Robin-Robin coupling. Int. J. Num. Meth. Engrg., 2013.

[20] P. Causin, J.-F. Gerbeau, and F. Nobile. Added-mass effect in the design of partitioned algorithms for fluid-structure problems. Comput. Methods Appl. Mech. Engrg., 194(4244):4506-4527, 2005.

[21] D. Chapelle and K.J. Bathe. The Finite Element Analysis of Shells - Fundamentals. Springer, 2011. 2nd Edition.

[22] D. Chapelle and A. Ferent. Modeling of the inclusion of a reinforcing sheet within a 3D medium. Math. Models Methods Appl. Sci., 13(4):573-595, 2003.

[23] P. Crosetto, S. Deparis, G. Fourestey, and A. Quarteroni. Parallel algorithms for fluidstructure interaction problems in haemodynamics. SIAM J. Sci. Comput., 33(4):1598-1622, 2011.

[24] J. Degroote. Partitioned simulation of fluid-structure interaction. Arch. Comput. Method E., 20(3):185-238, 2013.

[25] P. Degroote, J. Bruggeman, R. Haelterman, and J. Vierendeels. Stability of a coupling technique for partitioned solvers in FSI applications. Comp. Es Struct., 86(23-24):22242234, 2008.

[26] E.N. Dvorkin and K.-J. Bathe. A continuum mechanics based four-node shell element for general nonlinear analysis. Engineering Computations, 1(1):77-88, 1984. 
[27] M. Eswaran, U.K. Saha, and D. Maity. Effect of baffles on a partially filled cubic tank: Numerical simulation and experimental validation. Computers \& Structures, 87(3-4):198205, 2009 .

[28] FELiScE. http://felisce.gforge.inria.fr

[29] M. A. Fernández. Coupling schemes for incompressible fluid-structure interaction: implicit, semi-implicit and explicit. SëMA J., 55(1):59-108, 2011.

[30] M. A. Fernández. Incremental displacement-correction schemes for the explicit coupling of a thin structure with an incompressible fluid. C. R. Math. Acad. Sci. Paris, 349(7-8):473-477, 2011.

[31] M. A. Fernández. Incremental displacement-correction schemes for incompressible fluidstructure interaction: stability and convergence analysis. Numer. Math., 123(1):21-65, 2013.

[32] M. A. Fernández, J.F. Gerbeau, and C. Grandmont. A projection semi-implicit scheme for the coupling of an elastic structure with an incompressible fluid. Int. J. Num. Meth. Engrg., 69(4):794-821, 2007.

[33] M. A. Fernández and M. Moubachir. A Newton method using exact Jacobians for solving fluid-structure coupling. Comp. \& Struct., 83:127-142, 2005.

[34] M. A. Fernández and J. Mullaert. Convergence and error analysis for a class of splitting schemes in incompressible fluid-structure interaction. IMA J. Numer. Anal., 2015.

[35] M. A. Fernández, J. Mullaert, and M. Vidrascu. Explicit Robin-Neumann schemes for the coupling of incompressible fluids with thin-walled structures. Comput. Methods Appl. Mech. Engrg., 267:566-593, 2013.

[36] M. A. Fernández, J. Mullaert, and M. Vidrascu. Generalized Robin-Neumann explicit coupling schemes for incompressible fluid-structure interaction: stability analysis and numerics. Internat. J. Numer. Methods Engrg., 101(3):199-229, 2015.

[37] Miguel A. Fernández, Mikel Landajuela, and Marina Vidrascu. Fully decoupled timemarching schemes for incompressible fluid/thin-walled structure interaction. Journal of Computational Physics, 297(0):156 - 181, 2015.

[38] C. Förster, W.A. Wall, and E. Ramm. Artificial added mass instabilities in sequential staggered coupling of nonlinear structures and incompressible viscous flows. Comput. Methods Appl. Mech. Engrg., 196(7):1278-1293, 2007.

[39] N. Gaddum, O. Holub, A. Hessenthaler, R. Sinkus, and D. Nordsletten. Benchmark experiment for validation of fluid-structure interaction algorithms. In 4 th International Conference on Computational \& Mathematical Biomedical Engineering (CMBE15), Cachan (France), July 2015.

[40] M.W. Gee, U. Küttler, and W. Wall. Truly monolithic algebraic multigrid for fluid-structure interaction. Int. J. Numer. Meth. Engng., 85(8):987-1016, 2011.

[41] A. Geist, A. Beguelin, J. Dongarra, W. Jiang, R. Manchek, and V. Sunderam. PVM-parallel virtual machine: a users' guide and tutorial for networked parallel computing. MIT Press, Cambridge, Mass., 1994. 
[42] J.-F. Gerbeau and M. Vidrascu. A quasi-Newton algorithm based on a reduced model for fluid-structure interactions problems in blood flows. Math. Model. Num. Anal., 37(4):631648, 2003.

[43] J.-F. Gerbeau, M. Vidrascu, and P. Frey. Fluid-structure interaction in blood flows on geometries based on medical imaging. Comp. \& Struct., 83(23):155-165, 2005.

[44] G. Guidoboni, R. Glowinski, N. Cavallini, and S. Canic. Stable loosely-coupled-type algorithm for fluid-structure interaction in blood flow. J. Comp. Phys., 228(18):6916-6937, 2009 .

[45] M. Heil and A.L. Hazel. Fluid-structure interaction in internal physiological flows. In Annual review of fluid mechanics. Volume 43, 2011, volume 43 of Annu. Rev. Fluid Mech., pages 141-162. Annual Reviews, 2011.

[46] G. Hou, J. Wang, and A. Layton. Numerical methods for fluid-structure interaction-a review. Commun. Comput. Phys., 12(2):337-377, 2012.

[47] D. Kamensky, M.-C. Hsu, D. Schillinger, J.A. Evans, A. Aggarwal, Y. Bazilevs, M.S. Sacks, and T.J.R. Hughes. An immersogeometric variational framework for fluid-structure interaction: Application to bioprosthetic heart valves. Comput. Methods Appl. Mech. Engrg., 284:1005-1053, 2015.

[48] U. Küttler, M.W. Gee, C. Förster, A. Comerford, and W.A. Wall. Coupling strategies for biomedical fluid-structure interaction problems. Int. J. Numer. Meth. Biomed. Engng., 26(3-4):305-321, 2009.

[49] P. Le Tallec. Domain decomposition methods in computational mechanics. In J. Tinsley Oden, editor, Computational Mechanics Advances, volume 1 (2), pages 121-220. NorthHolland, 1994.

[50] P. Le Tallec and J. Mouro. Fluid structure interaction with large structural displacements. Comput. Meth. Appl. Mech. Engrg., 190:3039-3067, 2001.

[51] P. Le Tallec and M. Vidrascu. Solving large scale structural problems on parallel computers using domain decomposition techniques. In M. Papadrakakis, editor, Parallel Solution Methods in Computational Mechanics, chapter 2, pages 49-82. J. Wiley, 1997.

[52] J. Liu, R.K. Jaiman, and P.S. Gurugubelli. A stable second-order scheme for fluid-structure interaction with strong added-mass effects. J. Comput. Phys., 270:687-710, 2014.

[53] R. Löhner, J. R. Cebral, C. Yang, J. D. Baum, E. L. Mestreau, and O. Soto. Extending the range and applicability of the loose coupling approach for FSI simulations. In H.-J. Bungartz and M. Schäfer, editors, Fluid-Structure Interaction, volume 53 of Lecture Notes in Computational Science and Engineering, pages 82-100. Springer Berlin Heidelberg, 2006.

[54] M. Lombardi, N. Parolini, A. Quarteroni, and G. Rozza. Numerical simulation of sailing boats: Dynamics, FSI, and shape optimization. In G. Buttazzo and A. Frediani, editors, Variational Analysis and Aerospace Engineering: Mathematical Challenges for Aerospace Design, Springer Optimization and Its Applications, pages 339-377. Springer, 2012.

[55] M. Lukacova-Medvid'ovaa, G. Rusnakovaa, and A. Hundertmark-Zauskovaa. Kinematic splitting algorithm for fluid-structure interaction in hemodynamics. Comput. Methods Appl. Mech. Engrg., 265(1):83-106, 2013. 
[56] J. Mandel. Balancing domain decomposition. Comm. Numer. Methods Engrg., 9(3):233-241, 1993.

[57] H. Melbø and T. Kvamsdal. Goal oriented error estimators for Stokes equations based on variationally consistent postprocessing. Comput. Methods Appl. Mech. Engrg., 192(5-6):613633, 2003.

[58] Mahdi Esmaily Moghadam, Yuri Bazilevs, Tain-Yen Hsia, Irene E Vignon-Clementel, Alison L Marsden, et al. A comparison of outlet boundary treatments for prevention of backflow divergence with relevance to blood flow simulations. Computational Mechanics, 48(3):277$291,2011$.

[59] P. Moireau, C. Bertoglio, N. Xiao, C.A. Figueroa, C.A. Taylor, D. Chapelle, and J.-F. Gerbeau. Sequential identification of boundary support parameters in a fluid-structure vascular model using patient image data. Biomech. Model. Mechanobiol., 12(3):475-496, 2013.

[60] P. Moireau, N. Xiao, M. Astorino, C. A. Figueroa, D. Chapelle, C. A. Taylor, and J-F. Gerbeau. External tissue support and fluid-structure simulation in blood flows. Biomech. Model. Mechanobiol., 11:1-18, 2012.

[61] R.L. Muddle, M. Mihajlović, and M. Heil. An efficient preconditioner for monolithicallycoupled large-displacement fluid-structure interaction problems with pseudo-solid mesh updates. J. Comput. Phys., 231(21):7315-7334, 2012.

[62] F. Nobile, M. Pozzoli, and C. Vergara. Time accurate partitioned algorithms for the solution of fluidstructure interaction problems in haemodynamics. Comput. Fluids, 86:470-482, 2013.

[63] F. Nobile, M. Pozzoli, and C. Vergara. Inexact accurate partitioned algorithms for fluidstructure interaction problems with finite elasticity in haemodynamics. J. Comput. Phys., 273:598-617, 2014.

[64] M.P. Païdoussis, S.J. Price, and E. de Langre. Fluid-structure interactions: cross-flowinduced instabilities. Cambridge University Press, 2011.

[65] J. Pereira Gomes, S. Yigit, H. Lienhart, and M. Schäfer. Experimental and numerical study on a laminar fluid-structure interaction reference test case. J. Fluids Struct., 27(1):43-61, 2011.

[66] C. Pozrikidis. Computational hydrodynamics of capsules and biological cells. Chapman \& Hall/CRC Mathematical and Computational Biology. CRC Press, 2010.

[67] A. Quaini and A. Quarteroni. A semi-implicit approach for fluid-structure interaction based on an algebraic fractional step method. Math. Models Methods Appl. Sci., 17(6):957-983, 2007.

[68] T. Richter. A monolithic geometric multigrid solver for fluid-structure interactions in ale formulation. Int. J. Num. Meth. Engrg., 104(5):372-390, 2015.

[69] Th. Richter and Th. Wick. Finite elements for fluid-structure interaction in ALE and fully eulerian coordinates. Comput. Methods Appl. Mech. Engrg., 199(41-44):2633-2642, 2010.

[70] B. Smith, P. Bjorstad, and W. Gropp. Domain Decomposition: Parallel Multilevel Methods for Elliptic Partial Differential Equations. Cambridge University Press, 1996. 
[71] K Stein, T Tezduyar, and R Benney. Mesh moving techniques for fluid-structure interactions with large displacements. Journal of Applied Mechanics, 70(1):58-63, 2003.

[72] E. W. Swim and P. Seshaiyer. A nonconforming finite element method for fluid-structure interaction problems. Comput. Methods Appl. Mech. Engrg., 195(17-18):2088-2099, 2006.

[73] E.W. Swim and P. Seshaiyer. A nonconforming finite element method for fluid-structure interaction problems. Comput. Methods Appl. Mech. Engrg., 195(17-18):2088-2099, 2006.

[74] S. Sy and C.M. Murea. A stable time advancing scheme for solving fluid-structure interaction problem at small structural displacements. Comput. Methods Appl. Mech. Engrg., 198(2):210-222, 2008.

[75] K. Takizawa and T.E. Tezduyar. Computational methods for parachute fluid-structure interactions. Arch. Comput. Methods Eng., 19:125-169, 2012.

[76] T.E. Tezduyar. Stabilized finite element formulations for incompressible flow computations. In Advances in applied mechanics, Vol. 28, volume 28 of Adv. Appl. Mech., pages 1-44. Academic Press, Boston, MA, 1992.

[77] S. Turek, J. Hron, M. Razzaq, H. Wobker, and M. Schäfer. Numerical benchmarking of fluidstructure interaction: A comparison of different discretization and solution approaches. In H.-J. Bungartz, M. Mehl, and M. Schäfer, editors, Fluid Structure Interaction II, volume 73 of Lecture Notes in Computational Science and Engineering, pages 413-424. Springer Berlin Heidelberg, 2010.

[78] E. H. van Brummelen. Added mass effects of compressible and incompressible flows in fluid-structure interaction. J. Appl. Mech., 76(2):021206-7, 2009.

[79] E. H. van Brummelen. Partitioned iterative solution methods for fluid-structure interaction. Int. Jour. Num. Meth. Fluids, 65(1-3):3-27, 2011.

[80] E. H. van Brummelen, K. G. van der Zee, V. V. Garg, and S. Prudhomme. Flux evaluation in primal and dual boundary-coupled problems. J. Appl. Mech., 79(1):010904-010904, 2011.

[81] J. Young and S. Mitran. A numerical model of cellular blebbing: A volume-conserving, fluid-structure interaction model of the entire cell. J. Biomech., 43(2):210-220, 2010. 


\section{Contents}

\begin{tabular}{llr}
\hline 1 & Introduction & 3 \\
\hline
\end{tabular}

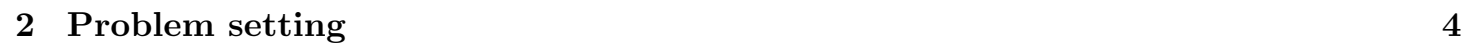

2.1 Geometry . . . . . . . . . . . . . . . . . . . . . 4

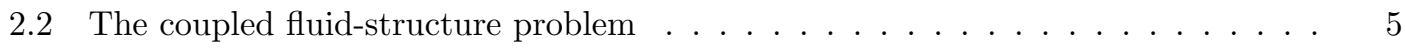

2.2.1 Coupling with 3D solid model . . . . . . . . . . . . . . . . . . . . . . 5

2.2 .2 Coupling with thin-walled solid model . . . . . . . . . . . . . . . . 6

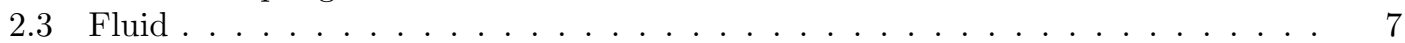

2.3 .1 Phase I experiment . . . . . . . . . . . . . . . . . . . . . 7

2.3 .2 Phase II experiment . . . . . . . . . . . . . . . . . . . . . . . . . . . 7

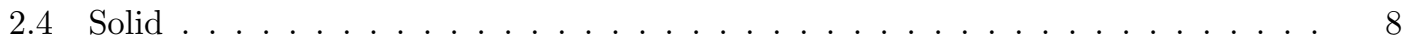

2.4 .1 Solid parameters estimation . . . . . . . . . . . . . . . 9

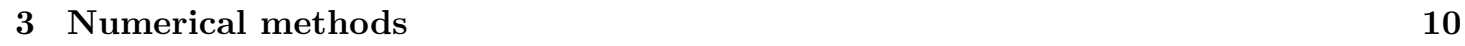

3.1 Time discretization: coupling schemes . . . . . . . . . . . . . . . . . . . . . . . . 10

3.1.1 Strongly coupled schemes . . . . . . . . . . . . . . . . . . . . 11

3.1.2 Projection based semi-implicit coupling schemes . . . . . . . . . . . . . . 11

3.1.3 Explicit coupling schemes . . . . . . . . . . . . . . . . . . . . 12

3.2 Space discretization $\ldots \ldots \ldots \ldots \ldots \ldots \ldots$. . . . . . . . . . . . . . . . . . . . . . . . . . . . . . . . . . .

3.3 Mesh update technique . . . . . . . . . . . . . . . . . . . . 16

4 Numerical results and comparison with experimental data 17

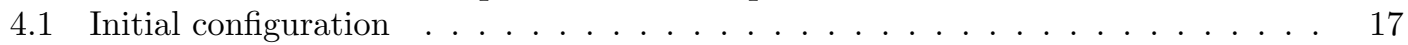

4.2 Phase I experiment . . . . . . . . . . . . . . . . . . . . . . . . . . . . . . 18

4.3 Phase II experiment $\ldots \ldots \ldots \ldots \ldots \ldots \ldots$

\begin{tabular}{|lr}
5 Conclusion & 24 \\
\hline
\end{tabular} 


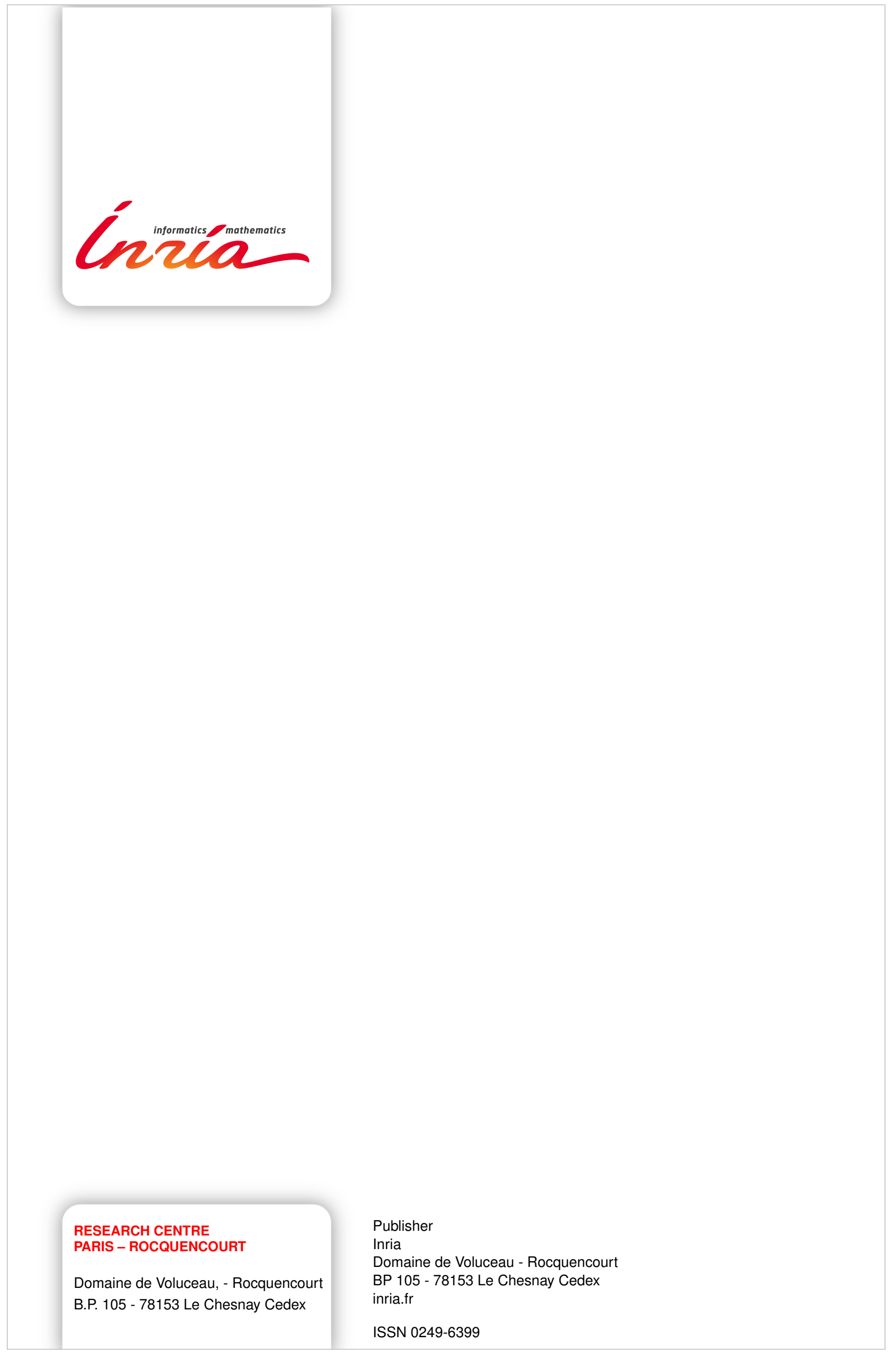

